\title{
Heterologous Expression of Cyanobacterial PCS Confers Augmented Arsenic And Cadmium Stress Tolerance and Higher Artemisinin In Artemisia Annua Hairy Roots.
}

Neha Pandey

University of Allahabad

Krishna Kumar Rai

Banaras Hindu University

Sanjay Kumar Rai

Rajendra Agricultural University: Dr Rajendra Prasad Central Agricultural University

Shashi Pandey-Rai ( $\nabla$ shashi.bhubotany@gmail.com )

Institute of science https://orcid.org/0000-0002-6998-1594

\section{Research Article}

Keywords: Phytochelatin synthase, Artemisia annua, hairy root, Anabaena PCC 7120, Arsenic, cadmium.

Posted Date: February 18th, 2021

DOI: https://doi.org/10.21203/rs.3.rs-225658/v1

License: (c) (1) This work is licensed under a Creative Commons Attribution 4.0 International License.

Read Full License 


\section{Abstract}

The present study provides the first report of heterologous expression of phytochelatin synthase from Anabaena PCC 7120 (anaPCS) into the hairy roots of Artemisia annua. Transformed hairy roots of $A$. annua expressing anaPCS gene showed better tolerance to heavy metals viz., arsenic (As) and cadmium (Cd) owing to 143 and $191 \%$ more As and Cd accumulation respectively as compared to normal roots with a bioconcentration factor (BCF) of 9.7 and 21.1 for As and Cd respectively. Under As and Cd stresses, transformed hairy roots possessed significantly higher amounts of phytochelatins and thiols probably due to the presence of both AaPCS (Artemisia annua PCS) and anaPCS. In addition, artemisinin synthesis was also induced in transformed hairy roots under heavy metals stresses. In-silico analysis revealed the presence of conserved motifs in both $A a P C S$ and anaPCS sequences as well as structural modelling of PCS functional domain was conducted. Interaction of AaPCS and anaPCS proteins with $\mathrm{CdCl}_{2}$ and sodium arsenate gene ontology analysis gave insights to anaPCS functioning in transformed hairy roots of $A$. annua. The study provides transformed hairy roots of $A$. annua as an efficient tool for effective phytoremediation with added advantages of artemisinin extraction from hairy roots used for phytoremediation.

\section{Key Message}

Heterologous expression of Cyanobacterial PCS gene in Artemisia anuua hairy roots conferred enhanced heavy metal tolerance with high artemisinin which can serve dual purpose of phytoremediation and high artemisinin production.

\section{Introduction}

Arsenic (As) and cadmium (Cd) are among the major soil pollutants due to their high toxicity and long persistence. Both As and Cd are non-essential elements and adversely affect plant growth and development even at very low concentration in the soil.

The entry of As in plants is concentration gradient dependent. Out of four oxidation states of As [i.e., $\mathrm{As}(0), \mathrm{As}(-\mathrm{III}), \mathrm{As}(\mathrm{III})$ and $\mathrm{As}(\mathrm{V})]$ only arsenate $[\mathrm{As}(\mathrm{V})]$ and arsenite $[\mathrm{As}(\mathrm{III})]$ enter in to the plant roots via different transporter proteins. Among arsenate and arsenite forms of As, arsenite is more toxic (Khalid et al. 2017). As(V) is a chemical analogue of phosphorus hence uses $\mathrm{Pi}$ transporters, consisting of $\mathrm{Pi}$ transporter proteins (PHT), to enter in to the plant (LeBlanc et al. 2013). Inside the cell, As (V) is converted into As (III) by the action of arsenate reductase (Bleeker et al. 2006). On the other hand As(III) enters in to the plant cells through nodulin 26- like intrinsic proteins (NIPs) belonging to the class of plant aquaporins (Mukhopadhyay et al. 2014). Cd uptake takes place through several metal transporters such as ZIP (ZRTand IRT-like proteins) family, YSL (Yellow Stripe-Like) transporters and NRAMP (natural resistanceassociated macrophage protein) family of transporters. Cd is also known to enter plant symplast via passive transport or by using $\mathrm{Ca}^{2+}$ channels present on guard cell plasma membrane (Ismael et al. 2018). 
Once inside the plant, these heavy metals are immobilized, chelated and compartmentalized through metal-binding ligands which include organic acids, polypeptides or even small peptides. One such metalbinding polypeptide is phytochelatin (PC) which mitigate the harmful effects of heavy metal by chelation (Zhang et al. 2013). PCs are enzymatically synthesized from glutathione by phytochelatin synthase (PCS). Heavy metals significantly elevate the biosynthesis of PCs. Metal ions including both anions (for example arsenate) and cations (for example cadmium) tend to increase the level of PCs (Cobbett 2001). Cloning of PCS gene has been achieved in many plant species such as Arabidopsis, rice and wheat and overexpression of PCS has been linked with greater heavy metal tolerance (Vatamaniuk et al. 1999).

Plant's potential to uptake heavy metals can be easily evaluated by calculating bioconcentration factor. Plants with a value of bioconcentration factor $>1$ for any heavy metal has been suggested to be categorized as bio-accumulators and could be beneficial for phytoextraction (Usman et al 2013).

Hairy roots are formed after infection with a gram-negative bacterium Agrobacterium rhizogenes, which transfers its T-DNA region (transfer DNA) into the host cell, integrates with the plant genome and expresses. Cloning and expression of recombinant proteins can be achieved by using many systems such as bacterial, yeast expression system or mammalian cells. However, each of them poses some or other limitations such as risks of viral infections in bacterial systems, toxic molecules in the mammalian system or high production cost (Gutierrez-Valdes et al. 2020). For a couple of decades plants systems are being used for several advantages including low cost. Hairy root cultures have emerged as a potential expression system with low maintenance cost and high efficiency. As hairy roots are derived from the wounded region of plants, they possess the genetic makeup of the plant part from which they are derived and hence do not behave like a true root. This property makes hairy root cultures as an excellent expression system. Hairy roots have found their potential application for phytoremediation of heavy metals (Agostini et al. 2013).

Artemisia annua, popularly known for its antimalarial drug artemisinin, has been found to act as a hyperaccumulator of arsenic. In our previous studies (Kumari et al. 2017, Rai et al. 2014), A. annua was found to survive well even at high As-concentration and have hyperaccumulating capacity. Many heavy metal stresses including both As (Kumari et al. 2017) and Cd (Li et al. 2012) have been reported to induce biosynthesis of artemisinin. Various reports have been published for hairy root induction in $A$. annua for enhanced secondary metabolite production (Patra and Srivastava 2014). A. annua hairy root potential for phytoremediation of heavy metals has not been investigated. In the present investigation we have cloned a cyanobacterial PCS gene (from Anabaena PCC 7120) into hairy roots of $A$. annua. We hypothesize that transformed hairy roots overexpressing PCS gene can be used as an excellent tool for phytoremediation of arsenic and cadmium.

\section{Materials And Methods}

Plant material and hairy root induction 
Uniform seeds of Artemisia annua were surface sterilized in sodium hypochlorite ( $10 \% \mathrm{w} / \mathrm{v})$ solution for 15-20 min and washed three to four times in sterilized water. Seeds were then treated with ethanol (70\% $\mathrm{v} / \mathrm{v}$ ) for $30 \mathrm{sec}$ and were then allowed to germinate on Murashige and Skoog (MS) medium containing sucrose $(3 \% \mathrm{w} / \mathrm{v})$ at $\mathrm{pH} 5.8$. Germination initiated under the controlled condition of light and temperature $\left(16 / 8 \mathrm{~h} \mathrm{light/dark} \mathrm{regime} \mathrm{and} 25 \pm 2^{\circ} \mathrm{C}\right)$. The in-vitro raised plantlets were then used for induction of hairy roots by infecting the leaves / stem with Agrobacterium rhizogenes ATCC 15834 maintained in liquid YMB medium with O.D. at $600 \mathrm{~nm}$ ranged between 0.9 to 1.0 (Hooykass et al. 1977). The infected plantlets were then cultured on full strength MS medium for 2 days and then transferred to half-strength MS medium containing cefotaxime $\left(250 \mathrm{mg} \mathrm{l}^{-1}\right)$ under the same growth conditions. The plantlets were further sub-cultured by transferring them to new antibiotic supplemented medium for 2 weeks and finally the bacterial-free hairy roots were cultured on liquid MS medium. Hairy root cultures were incubated in dark inside the rotary shaker with constant rotation $(100 \mathrm{rpm})$ and were sub-cultured twice a month into the fresh medium.

For experiments, three tissue samples were taken: normal roots (which were derived from plantlets), hairy roots (derived from leaves by $A$. rhizogenes infection) and transformed hairy roots (also derived from leaves by $A$. rhizogenes infection and expressing anaPCS gene).

\section{Cyanobacterial strain and plasmids}

Anabaena sp. PCC 7120 was photoautotrophically grown in BG-11 medium (without any source of combined nitrogen) prepared in Tris buffer with a photoperiod of 14:10 h under light (day light fluorescent tubes) intensity of $72 \mu \mathrm{mol} \mathrm{m} \mathrm{m}^{-2} \mathrm{PAR}$ (Photosynthetically active radiation) at $24 \pm 2^{\circ} \mathrm{C}$ ) (Rippka et al. 1979). The E. coli strains DH5a was used for cloning and BL21 (Novagen) for overexpression. The cells possessing recombinant plasmid were transferred on fresh Luria-Bertani (LB) medium supplemented with $100 \mathrm{\mu gml}^{-1}$ ampicillin (Sambrook and Russell 2001). For cloning pGEX-5X-2 was used as a vector (Rai et al. 2008).

\section{Cloning and confirmation of anaPCS gene and transformation in $A$. annua hairy roots}

Total genomic DNA from Anabaena PCC7120 was isolated as per the standard CTAB method (Srivastava et al. 2007) and the region encoding phytochelatin synthase (alr0975, denoted as anaPCS in the manuscript) was amplified by using gene-specific primers having restriction sites for EcoRl and Notl (Chaurasia et al. 2008). The desired gene amplicon was then purified by the QIAquick gel extraction kit (Qiagen), cloned in pGEX-5X-2 vector (Novagen) containing EcoRI and Notl restriction sites and the resulting construct pGEX-5X-2-anaPCS was used to confirm anaPCS nucleotide sequence verification and expression analysis according to Rai et al. 2008. For heterologous expression analysis anaPCS gene was amplified using PF 5'-GCTCTAGACGATAGTTATGAAACTCTTTA-3', and PR 5'- 
GCAGAGCTCCTAATCTTGTGTTTTACTTAC-3', containing Xbal and Sacl restriction sites and inserted into a pBI121 (Clontech, USA) under the control of promoter CMV35S and as binary vector introduced into the A. rhizogenes ATCC 15834 by electroporation for further transformation of $A$. annua hairy roots and expression studies. The confirmation of the resulting recombinant vector pBI121-anaPCS was done by performing double digestion of the plasmid with $X b a l$ and $S a c 1$ enzymes and its sequencing.

\section{Plant stress treatments and measurement of arsenic and cadmium content}

To assess the role of anaPCS in conferring heavy metal tolerance, $A$. annua hairy roots transformed with pBI121-anaPCS, non-transformed hairy roots (empty vector), and normal roots were subjected to arsenic $(100 \mu \mathrm{M})$ and cadmium stress $(50 \mu \mathrm{M})$ using sodium arsenate $\left(\mathrm{Na}_{2} \mathrm{HAsO}_{4} .7 \mathrm{H}_{2} \mathrm{O}\right)$ and cadmium chloride $\left(\mathrm{CdCl}_{2}\right)$ respectively for 5 days. The treatments were divided in to three sets, set 1 with no treatment (control), Set 2 with $100 \mu \mathrm{M}$ of arsenate $\left(\mathrm{Na}_{2} \mathrm{HAsO}_{4} \cdot 7 \mathrm{H}_{2} \mathrm{O}\right)$ and set 3 with $\mathrm{CdCl}_{2}$. Each treatment set contains three groups of samples, untransformed hairy roots, transformed hairy roots and normal roots of the plant.

All the experiments were performed in triplicates. The accumulation of arsenic content in treated/nontreated hairy roots and normal roots was determined by the SEM-EDS method and was estimated by atomic absorption spectrometry (Perkin-Elmer A Analyst 600) as described by Kumari et al (2018).

\section{Estimation of phytochelatin synthase, reduced glutathione and thiol}

Phytochelatin content was determined as described earlier (Rai et al. 2011). For glutathione estimation $0.5 \mathrm{~g}$ tissue was homogenized in an extraction buffer containing sulphosalicylic acid ( $5 \% \mathrm{w} / \mathrm{v})$. The absorbance of the reaction mixture containing potassium phosphate buffer (100 mM, pH 7.0), DTNB (5'5'-dithiobis-2-nitrobenzoic acid) and tissue extract, was read at $412 \mathrm{~nm}$ (Anderson 1985). For total thiol estimation $0.5 \mathrm{~g}$ tissue was homogenized in extraction buffer containing ascorbate buffer $(20 \mathrm{mM})$ and EDTA $(20 \mathrm{mM})$ and after centrifugation at $12,000 \mathrm{x} \mathrm{g}$ for $15 \mathrm{~min}\left(4^{\circ} \mathrm{C}\right), 0.5 \mathrm{ml}$ supernatant was mixed with reaction mixture that contained Tris $-\mathrm{HCl}$ buffer $(200 \mathrm{mM})$ and DTNB $(10 \mathrm{mM})$. Reaction mixture developed yellow color after incubation for $20 \mathrm{~min}$ at $30^{\circ} \mathrm{C}$ and absorbance was read at $412 \mathrm{~nm}$ (Nagalakshmi and Prasad 2001).

\section{Measurement of antioxidative enzymes}

Fresh tissues $(500 \mathrm{mg}$ ) were homogenized with $5 \mathrm{ml}$ Tris-buffer $(50 \mathrm{mM}, \mathrm{pH}=7.0)$ that contains EDTA (2 $\mathrm{mM})$, PMSF (1 mM) and PVP (1\%). After centrifugation of homogenate at 12000xg for 15 minutes, immediately the supernatant was used for the assay. 
Antioxidative enzymes catalase, ascorbate peroxidase, glutathione reductase and peroxidases were measured as described earlier (Kumari et al. 2017, Pandey and Pandey-Rai 2014). All the spectrophotometric analysis was carried out using Hitachi U-2910 spectrophotometer.

\section{Expression analysis of rolB and PCS gene and artemisinin biosynthetic pathway genes}

Total RNA was isolated using Trizol reagent (Invitrogen) as per the manufacturer's recommendation. One microgram of total RNA was used as template for CDNA synthesis with cDNA synthesis kit (Bio-Rad Laboratories, USA) following the manufacturer's instructions.

RT-PCR analysis using primers listed in supplementary table 1, was performed by following the method described earlier (Pandey and Pandey-Rai 2014). All reactions were run in triplicate along with a negative control. Primer accuracy was tested using gDNA as positive control. PCR products were run on agarose gel and band intensities were measured using Quantity One software (BioRad) installed with Gel Doc 2000 system (BioRad).

\section{Artemisinin estimation}

Artemisinin was extracted and prepared according to Zhao and Zeng (1985) and Pu et al. (2009) with minor modifications. Approximately $100 \mathrm{mg}$ dry tissue was extracted in $20 \mathrm{ml}$ petroleum ether by ultrasonication (35 min). The extract was evaporated and dissolved in $1 \mathrm{ml}$ ethanol. After $1 \mathrm{~min}$ centrifugation at $10,000 \times \mathrm{g}, 0.1 \mathrm{ml}$ of the supernatant was treated with $0.4 \mathrm{ml}$ of $0.2 \%(\mathrm{w} / \mathrm{v}) \mathrm{NaOH}$ at 45 ${ }^{\circ} \mathrm{C}$ for $35 \mathrm{~min}$ and cooled to room temperature. The solution was acidified with $0.5 \mathrm{ml}$ of $0.05 \mathrm{M}$ acetic acid and filtered through a Millipore filter $(0.45 \mu \mathrm{m})$ before HPLC analysis. HPLC analysis conditions: Waters 600E HPLC system equipped with $150 \times 94.6$ mm Hypersil BDS C8 column; injected samples (10 $\mu \mathrm{l})$ were eluted with methanol / $0.01 \mathrm{M} \mathrm{Na}_{2} \mathrm{HPO}_{4}-\mathrm{NaH}_{2} \mathrm{PO}_{4}$ buffer $(\mathrm{pH} 7)(2: 3, \mathrm{v} / \mathrm{v})$ at $1 \mathrm{ml} \mathrm{min}{ }^{-1}$ and monitored at $260 \mathrm{~nm}$. The concentration of artemisinin was measured using the standard curve.

\section{In-silico analysis for comparative phylogeny, structural and functional analysis of anaPCS and AaPCS gene.}

The sequence of phytochelatin synthase (PCS) gene in A. annua (AaPCS) and Anabaena PCC7120 (anaPCS) was obtained using BLAST-P (http://blast.ncbi.nlm.nih.gov/Blast.cgi) by taking homology with the Arabidopsis phytochelatin synthase (AtPCS) gene (Locus: AAD41794.1) identified from TAIR (https://www.arabidopsis.org/). The sequences with maximum query coverage and percentage identity $(>90 \%)$ were selected for the phylogenetic tree construction and sequential characterization. The sequences were aligned by using Bio-Edit tool (Hall, 1999) and phylogenetic tree was constructed using MEGA 7 suite (Tamura et al., 2013). The result of BLAST-P search was further subjected to PDB database 
to evaluate the similarity indices of the sequences and confirmed by circos visualization tool (Krzywinski et al. 2009). All the identified probable PCS protein sequences were subjected to multiple sequence alignment analysis using CLC bio workbench and with the help of Clustal W (http://www.ebi.ac.uk/tools/msa/clustalw2/) for their sequential classification (Krzywinski et al. 2009). The functional domain regions occupied by PCS proteins were searched using InteProScanhttp://www.ebi.ac.uk/Tools/pfa/iprscan (Jones et al. 2014), NCBI CDD server http://www.ncbi.nlm.nih.gov/Structure/cdd/cdd.shtml (Marchler-Bauer et al. 2011) and ExPASy-Prosite scan http://prosite.expasy.org/scanprosite (de Castro et al. 2006). The occurrence of potential motifs in PCS protein in all the members was identified using MEME Suite 4.1.1.2 (Multiple EM for Motif Elicitation) (Bailey et al. 2006) by keeping the selection parameter to any number of repeats with motif width of 10 and 30 residues and by keeping a maximum number of motifs to 40 .

\section{Structural modelling and model validation}

The 3D homology modelling of identified protein sequences for PCS protein in A. annua and Anabaena PCC 7120 (AaPCS and anaPCS) was done by using template proteins available in protein database which were searched using BLAST-P programme of Protein Data Bank (www.rcsb.org/pdb/) (Berman et al. 2000). The result of BLAST-P analysis showed most significant and accurate templates for the modelling of functional domain structures of AaPCS and anaPCS proteins. Three most closely related proteins were selected for the homology modelling of AaPCS and anaPCS proteins using MODELLER module of Discovery Studio 3.0 (accelrys.com; Shahi et al. 2013). Five models were generated by the MODELLER using Crystal structure of anaPCS (template 1; PDB ID: 2BTW), Crystal structure of a putative C39-like peptidase from Bacillus anthracis (template 2; PDB ID: 3ERV) and Crystal structure of the Nterminal peptidase C39 like domain of the toxin secretion ATP-binding protein from Vibrio parahaemolyticus (template 3; PDB ID: 3B79). The model with lowest Discrete Optimized Protein Energy (DOPE) score was further refined for Ca traces by ModRefiner using two-step atomic level energy minimization module (Xu and Zhang 2011). The modelled proteins were then allowed to superimpose with each template using Superpose version 1.0 (Maiti et al. 2010) to access the topological details of the modelled proteins.

The predicted models were further evaluated qualitatively in terms of their geometric analysis, stereochemical orientation and backbone conformation patterns of nonbonded atomic interactions. For the backbone conformations, the predicted models were subjected to Ramachandran plot analysis for evaluating the backbone dihedral phi $(\varphi)$ and psi $(\Psi)$ angles using PROCHECK module of PDBSum server http://www.ebi.ac.uk/pdbsum/ (Laskowski et al. 2005) which was further attested by RAMPAGE server (Lovell et al. 2003). The models were also evaluated by a single model method using ProSA (Wiederstein and Sippl 2007), Qmean (Benkert et al 2009), RESPROX (Resolution by Proxy), ERRAT (Colovos and Yeates 1993). For the quantitative evaluation, the models were subjected to VADAR (Volume, Area, Dihedral Angle Reporter) analysis (Willard et al. 2003). The protein models generated for the functional 
domain were further submitted to an online repository of PMDB (Castrignano et al. 2006) to obtain the accession identities.

\section{Ligand modelling and protein docking}

The molecular docking studies between ligands (Cadmium chloride; $\mathrm{CdCl}_{2}$ and sodium arsenate; $\mathrm{Na}_{2} \mathrm{HAsO}_{4} .7 \mathrm{H}_{2} \mathrm{O}$ ) and the AaPCS and anaPCS protein sequences was carried out using Hex 8.0 molecular docking server (Macindoe et al. 2010) to reveal the probable binding modes for ligand compounds with in the active site of PCS protein. The ligands viz., $\mathrm{CdCl}_{2}$ and sodium arsenate were drawn in $2 \mathrm{D}$ structure using Chemdraw software (ChemDraw, CambridgeSoft Corporation, 875 Massachusetts Avenue, Cambridge, MA 02139, USA). For molecular docking study the 2D structure was converted to 3D structure and saved in PDB format. The protein and ligand interaction studies were performed following correlation criterion i.e. DARS+ Shape + Electro, FFT Mode-3D fast lite and grid range of 0.6 with Receptor: Ligand: Twist: Distance range of 180: 180: 360: 40. Several conformations of docked complexes were obtained, however, the docked complex having lowest binding energy values was analyzed by DS Studio 3.0 for identifying the key residues involved in the interaction with $\mathrm{CdCl}_{2}$ and sodium arsenate.

\section{Protein-protein interaction, structural and functional annotation}

The functional protein-protein interaction complex involved in signaling and metabolic pathways at both cellular, biological and molecular levels in AaPCS and AnaPCS proteins were searched through STRING server (Search Tool for the Retrieval of Interacting Genes/Proteins) database version $10.0 \mathrm{http}: / /$ string$\mathrm{db}$. org/ (Szklarczyk et al. 2015). The functional interactive sources were identified based on seven core shells such as co-expression, co-occurrence, neighbor, gene fusion, text-mining and experiments by setting confident score $(0.40)$ to default. The identified proteins were then sequentially analyzed for their structural and functional organization based on gene ontological (GO) terms using CATH server http://www.cathdb.info/ (Sillitoe et al. 2015) and FunFHMMER http://www.cathdb.info/search/byfunfhmmer (Das et al. 2016). The GO enrichment data obtained was further visualized using hypergeometric distribution test analysis of REVIGO web server http://revigo.irb.hr/ (Supek et al. 2011). The identified GO terms were further fetched to CELLO2GO web server http://cello.life.nctu.edu.tw/cello2go/ (Yu et al. 2014) for their probable subcellular localization.

\section{Active site prediction}

The prediction of transition metal binding sites and identification of potential residues involved in the making of the active sites with AaPCS and anaPCS was done using metapocket server http://metapocket.eml.org (Huang 2009). 


\section{Statistical analysis}

All the experiments were performed in three biological replications and subjected to analysis of variance (ANOVA). The mean differences were compared by performing Duncan's multiple range test (DMRT) using SPSS software (SPSS Inc., Version 20.0) and values at $P \leq 0.05$ were considered significant.

\section{Results}

\section{Induction and establishment of normal roots, hairy roots and transformed hairy root}

Plantlets of $A$. annua were successfully established under in-vitro conditions (supplementary fig.1) and produced visible roots after 15 days of inoculation onto the MS medium. Infection by $A$. rizogenes carrying binary vector (pBI121-anaPCS) and co-culturing of infected $A$. annua leaves has resulted in transformed hairy root (containing anaPCS gene) induction after 3 week of inoculation at a relative transformation frequency of $71 \%$. Normal hairy roots not carrying anaPCS gene were also obtained in 15 days by infecting $A$. annua leaves with $A$. rhizogenes and co-culturing leaf discs. Presence of rol $B$ gene in normal hairy roots and transformed hairy roots (with anaPCS) confirmed their origin after $A$. rhizogenes infection. The rolB gene was absent in normal roots (Fig. 1).

\section{Transformed hairy roots expressing anaPCS show enhanced tolerance (accumulation) to heavy metal stresses}

In $A$. annua, hairy roots accumulated very high amount of $\mathrm{As}$ and $\mathrm{Cd}$ as compared to normal roots under As and Cd stress respectively (Fig. 2). However, hairy roots expressing anaPCS gene accumulated even higher amount of both heavy metals as compared to non-transformed hairy roots. Under As-stress, nontransformed hairy roots and transformed hairy roots accumulated $91.3 \%$ and $143.4 \%$ higher amount of As respectively as compared to normal roots of $A$. annua. Among hairy roots, $72.7 \%$ greater As accumulation was observed in transformed hairy roots expressing anaPCS gene. Similar trend was observed for Cd-accumulation, where non-transformed hairy roots and transformed hairy roots accumulated $36 \%$ and $191 \%$ more $\mathrm{Cd}$ as compared to normal roots. Further among hairy roots, transformed hairy roots showed 55.4 \% greater $\mathrm{Cd}$ accumulation as compared to non-transformed hairy roots.

The bioconcentration factor (BCF) for As was found to be 3.71, 5.71 and 9.7 for normal roots, nontransformed hairy roots and transformed hairy roots respectively (Fig. 3). Similarly, for Cd, bioconcentration factor was 9.4, 12.7 and 21.1 for normal roots, non-transformed hairy roots and transformed hairy roots respectively. The results showed that bioconcentration of $\mathrm{Cd}$ is higher than As and hairy roots expressing anaPCS gene have greater BCF for both As and Cd as compared to nontransformed hairy roots. 
Normal roots, hairy roots (with only rol gene) and transformed hairy roots (with rol and anaPCS gene) have morphological differences in terms of average weight and average weight increase and has been listed in supplementary table 6 . As visible in the supplementary figure 10, branching pattern is also different among normal, hairy and transformed hairy roots. Transformed hairy roots (with anaPCS gene) had more branching as compared to normal and hairy roots (without anaPCS gene).

\section{Phytochelatins and phytochelatin synthase expression}

Heavy metal stresses enhanced the phytochelatin content in all the tissue samples however the concentration was significantly more in hairy roots rather than normal roots (Fig. 4A). As-stress boosted phytochelatin content up to $28.3 \%, 44.7 \%$ and $14.7 \%$ in normal roots, hairy roots and transformed hairy roots respectively as compared to their respective control. Cd-stress enhanced phytochelatin level up to $25 \%, 35.7 \%$ and $9.52 \%$ in normal, hairy roots and transformed hairy roots respectively in comparison to their respective control. Although the stress-induced percent increase of phytochelatin was comparatively less prominent in transformed hairy roots, the amount of phytochelatin was more. Under conditions of As and Cd stress, transformed hairy roots expressing anaPCS gene was shown to contain $35.3 \%$ and $37.7 \%$ more phytochelatin as compared to non-transformed hairy roots.

The expression of phytochelatin synthase was measured through RT-PCR. Expression of anaPCS was observed only in transformed hairy roots and because normal roots and hairy roots were not carrying anaPCS gene copy, no expression was observed in them (Fig. 4B). Expression of anaPCS was reported to be induced up to 2.1 and 2.3-folds under As and Cd treatment respectively as compared to control. Expression of AaPCS (Artemisia annua PCS) was observed in all the tissue samples. AaPCS expression was significantly induced under As and $C d$ stress in hairy roots and transformed hairy roots however, no significant elevation in AaPCS was observed in normal roots under heavy metal stress conditions.

\section{Thiol and GSH level in normal roots, hairy roots and transformed hairy roots under As and Cd stress.}

In control samples (without heavy metal stress) of normal roots, hairy roots and transformed hairy roots, thiol content was found to 11.2, 12.2 and $21.1 \mu^{M^{-1}} \mathrm{FW}$ (Fig. 5A). Under condition of As-stress, thiol content was elevated up to $\mathbf{4 3 . 7 5} \%, 59.83 \%$ and $87.2 \%$ in normal roots, hairy roots and transformed hairy roots respectively, as compared to their respective controls. However, under Cd-stress, the percentage increase was $39.2 \%, 38.5 \%$ and $52.6 \%$ for normal roots, hairy roots and transformed hairy roots respectively, as compared to their respective control. Among hairy roots, thiol content was increased up to $102.5 \%$ under As-stress and $90.5 \%$ under Cd-stress in transformed hairy roots as compared to nontransformed hairy roots.

In contrast to thiol content, GSH level (Fig. 5B) was reduced under both heavy metal stresses in all tissue samples. Under As-stress $6.8 \%, 34.4 \%$ and $50.6 \%$ reduction in GSH level were observed in normal roots, 
hairy roots and transformed hairy roots respectively as compare to their respective control. Compared with the control, Cd-stress dropped GSH level up to $9.8 \%, 32.2 \%$ and $45.7 \%$ in normal roots, hairy roots and transformed hairy roots respectively. As compared to non-transformed hairy roots, transformed hairy roots expressing anaPCS gene contained $30.5 \%$ and $26.2 \%$ lower levels of GSH under As and Cd stress respectively.

\section{Stress-induced antioxidative enzymes in normal roots, hairy roots and transformed hairy roots}

Variable activities of four antioxidative enzymes were measured under heavy metal stress in all tissue samples (Fig. 6 A to D). Under As-stress, catalase activity was reported to be induced up to $20 \%, 12.5 \%$ and $20 \%$ in normal roots, hairy roots and transformed hairy roots respectively as compared to control. Similarly, Cd-stress increased catalase activity up to $26.6 \%, 12.5 \%$ and $13.3 \%$ in normal roots, hairy roots and transformed hairy roots as compared to control. Although catalase activity under stresses was higher than control, their activity was similar among all three tissues. Transformed hairy roots did not show any significant change in catalase activity under heavy metals stresses in $A$. annua.

Under As- stress APX activity was enhanced up to $21.9 \%, 67.6 \%$ and $34.7 \%$ in normal roots, hairy roots and transformed hairy roots respectively as compared to control. Under Cd-stress APX activity was reported to be $17.4 \%, 72.3 \%$ and $5.37 \%$ in normal roots, hairy roots and transformed hairy roots respectively compared with the control. In comparison to non-transformed hairy roots, transformed hairy roots expressing anaPCS gene showed $31.9 \%$ greater APX activity under As-stress, however, Cd-stressed transformed hairy root did not show any significant increment in APX activity as compared to nontransformed hairy roots.

Peroxidase activity was also enhanced up to $150 \%, 139.4 \%$ and $101.3 \%$ in As-stressed normal roots, hairy roots and transformed hairy roots respectively as compared to their respective control. Increment of $130.7 \%, 153.5 \%$ and $90.5 \%$ in peroxidase activity was reported in Cd-stressed normal roots, hairy roots and transformed hairy roots respectively as compared to control.

Stress-induced increment in GR activity was more pronounced in transformed hairy roots than nontransformed hairy roots. As-stress induced GR activity up to $5.5 \%, 18.6 \%$ and $151.7 \%$ in normal roots, hairy roots and transformed hairy roots respectively as compared to control. Similarly, Cd-stress enhanced GR activity up to $13.4 \%, 15.2 \%$ and $93.1 \%$ in normal roots, hairy roots and transformed hairy roots respectively as compared to control. Transformed hairy roots expressing anaPCS gene showed $108.5 \%$ and $64.7 \%$ greater GR activity under As and Cd stress respectively.

\section{Artemisinin content and expression of artemisinin biosynthetic pathway genes}


Artemisinin level was found to be more in hairy roots than in normal roots in both normal and stressed conditions (Fig. 7A). Under As-stress, artemisinin level was enhanced up to $50 \%, 33.3 \%$ and $16.6 \%$ in normal roots, hairy roots and transformed hairy roots respectively. On the other hand, Cd-stress boosted artemisinin level up to $45.41 \%, 25 \%$ and $22.2 \%$ in normal roots, hairy roots and transformed hairy roots respectively as compared to control. Fig. $7 \mathrm{~B}$ is showing the expression pattern of four key artemisinin biosynthetic pathway genes viz., ADS, CYP71AV1, DBR2 and $A L D H 1$, in all tissue samples. All the four biosynthetic pathway genes were found to be significantly overexpressed in transformed hairy roots as compared to normal roots and hairy roots (non-transformed). Under As-stress, ADS expression was 7 and 6.4-folds more than normal roots and hairy roots respectively. Similarly, in transformed hairy roots Cdstress enhanced ADS expression up to 2.8 and 3.2 -folds as compared to normal roots and hairy roots respectively. Similar overexpression patterns were observed for CYP71AV1, DBR2 and ALDH1 as well.

\section{Database mining and phylogenomic analysis of $A a P C S$ and anaPCS}

To find A. annua and Anabaena PCC 7120 PCShomolog, BLAST-P search was performed using the Arabidopsis PCS gene (AAD41794.1) which led to the identification of 06 genes with high homology. The sequences (PWA96196.1) comprehending the highest query coverages (100\%) and identity (73\%) with the target sequence were selected for further studies. Comparative phylogenomic analysis of AaPCS genes performed by constructing a phylogenetic tree based on UPGMA (Fig. 8A) and maximum parismony methods (Fig. 8B) revealed monophyletic origin with S. tuberosum (CAD68110.1) and with $O$. sativa (AA013349.2) respectively. In contrasts, other members viz., B. juncea (CAC37692.1) and Anabaena PCC 7120 (BAD10973.1) were observed to form a separate clade. The result of phylogenetic analysis among different plant species revealed homology of AaPCS with other homolog and ortholog members, established the evolutionary relationship between them and predicted that AaPCS is the closest homologue of StPCS.

\section{Functional sites identification and domain analysis}

The functional signature sequences underlying $A a P C S$ and anaPCS were identified by PROSITE analysis which validated and confirmed that the query sequences of both AaPCS and anaPCS belongs to Papainlike cysteine peptidase superfamily. The position of functional signature sequences constituting phytochelatin catalytic domain from N-terminal end (NTD) was also retrieved. The results of InteProScan analysis further confirm the presence of phytochelatin catalytic domain from $\mathrm{N}$-terminal domain in each AaPCS and anaPCS. In AaPCS, the N-terminal phytochelatin catalytic domain (NTD) prevailed from 7 to 234 amino acid residue where core signature sequences were occupied between $\mathrm{Tyr}^{7}$ and $\operatorname{Arg}^{234}$ (Supplementary Fig. 2) whereas in anaPCS, the N-terminal phytochelatin catalytic domain (NTD)

existed between 25 to 237 amino acid residues consisting functional core residues in between $\mathrm{Leu}^{25}$ and Lys $^{237}$ (Supplementary Fig. 3).The multiple sequence alignment (Supplementary fig. 4 ) results depicted 
conserved residues within the N-terminal phytochelatin catalytic domain (NTD) of the protein along with some insertion and deletion of the amino acid residues at certain positions that may have led to the sequence divergence in other homologs and orthologs, thus delineating their ancestral evolutionary origin. Further, circus visualization tools confirmed the ancestral evolutionary similarity and differences across the members of phytochelatin synthase family (Fig. 9). At $50 \%$, cut-off values, AaPCS displayed close relation with $S$. tuberosum and $A$. thaliana whereas anaPCS did not found in relation with any of the other homolog or ortholog members.

\section{Conserved motif analysis}

To comprehend conservation and diversification of PCS proteins in A. annua, putative functionally conserved network elements (motifs) of AaPCS were predicted using MEME software which predicted seven different motifs (Fig. 10). As anticipated, MEME motif analysis revealed that all the members in phylogenetic tree shared conjoint motif distribution pattern, signifying that PCS proteins within the same subclades might be involved in similar phytochelatin catalytic function. In our results, the motif scan analysis revealed significant motifs that comprised phytochelatin catalytic domain with in PCS proteins and statistical significance of the motifs were evaluated in terms of their E-value that speculate their adequate patterns and $p$-value that conjectures how substantially each pattern correlate with the motif. The N-terminal catalytic domain (NTD) in AaPCS was represented by motif 3 (MAMASLYRRLPSPPAIDF; $p$-value 1.3e-23) and propensity of the amino acids constituting this motif was revealed based on size of the alphabets that correspond to amino acids. The motif distribution pattern revealed via motif scan analysis unveil commonalities in $A$. annua, $A$. thaliana, S. tuberosum and B. juncea (motif 3: MAMASLYRRLPSPPAIDF; $p$-value 1.7e-65). Furthermore, the motif results showed the presence of additional 1 motif in $O$. sativa (RRLFRCAQAQ; $p$-value 2.9e-11) and additional 2 motifs in Anabaena PCC 7120 (QVARQGLTLD; $p$-value 1.0e-12 and PNLIGFNENE; $p$-value 1.1e-9) may have led to sequence divergence which could have been reason behind separate clustering of both OSPCS and anaPCS in the phylogenetic tree constructed in the present study.

\section{Structural modelling of PCS functional domain}

In the present study, the N-terminal catalytic domain of both AaPCS and anaPCS was modelled using relevant templates chosen based on crystal resolution, sequence similarity and completeness. $A$ total of five models each for AaPCS (Fig. 11A) and anaPCS (Fig. 11B) were generated by MODELLER module of DS Modeller (Supplementary table 2). In the present study, the models retaining lowest RMSD value corresponding to $\mathrm{Ca}$ atoms within the template crystal structures and minimum electrostatic energy (calculated) were considered as good stable models which were selected for further interactions with $\mathrm{CdCl}_{2}$ and $\mathrm{Na}_{3} \mathrm{AsO}_{4}$ (sodium arsenate) ligands modelled using ChemDraw tool. The predicted models were submitted to PMDB database and assigned PMDB IDs for our predicted structures of AaPCS (PM0083228) and anaPCS (PM0083229). 


\section{Model evaluation and validation}

The three-dimensional models both for $A a P C S$ and anaPCS were generated by satisfying all spatial restraint using Modeller Discovery Studio Client 3.0. Five sets of models each for AaPCS and anaPCS were arranged based on their Discrete Optimized Protein Energy (DOPE) and molecular Probability Density Functions (PDFs). The models (both for $A$. annua and Anabaena PCC7120) exhibiting minimum DOPE scores and optimum energy values were selected for further validation using various statistics. The model proteins were subjected to Ramachandran plot analysis using RAMPAGE and PDBSum servers to assess the behavior of amino acid residues. The RAMPAGE statistics shows the presence of $95.4 \%$ residues in most favored region with $3.2 \%$ in additionally allowed region and $1.4 \%$ in outlier region for AaPCS whereas for anaPCS the statistics revealed the occurrence of $98.2 \%$ residues in most favored region with $1.8 \%$ in additionally allowed region and $0.0 \%$ in outlier region. Further, VADAR analysis revealed that both the modelled proteins viz., AaPCS and anaPCS existed as helix type structure 68 \& 80 (30 \& 36\%) with $57 \& 59(25 \& 26 \%)$ and $96 \& 80$ (43 and 37\%) coil type configuration having mean $\mathrm{H}$ bond energy $(-1.6(\mathrm{sd}=1.1)$ against the expected values $(-2.0 \mathrm{sd}=0.8)$. Furthermore, PROCHECK evaluation (Supplementary fig. 6) revealed the occurrence of $87.6 \%$ (AaPCS) and $94.5 \%$ (anaPCS) residues in most favored region (A, B, L), with 8.8\% (AaPCS) and 5.0\% (anaPCS) in additionally allowed region $(a, b, I, p)$. A protein model is generally considered as a good quality model if it has $90 \%$ of the residues in most favored region which corroborates the results of the present study. Additionally, ProSA results also validated the results of RAMPAGE and PROCHECK analysis and confirmed that both the modelled proteins were precisely similar to template proteins. Moreover, RESPROX and QMEAN analysis confirmed the reliability of our predicted models by generating a composite score based on the template's proteins (Supplementary table 3).

\section{Protein-ligand interaction}

To further explore the core amino acid residues of AaPCS and anaPCS involved in the binding with the ligand, molecular docking studies were performed for the possible investigation of binding mode of ligand viz., cadmium chloride $\left(\mathrm{CdCl}_{2}\right)$ to the given receptors. The ligand viz., $\mathrm{CdCl}_{2}$ was modelled using the ChemDraw tool and was docked with the modelled proteins through HexDock server. In the present study, most reliable protein models of AaPCS and anaPCS were docked and stability of the docked complex was measured in terms of binding energy. For AaPCS the most stable docked complex has the binding energy of $-134.58 \mathrm{Kcal} / \mathrm{mol}$ whereas in case of anaPCS the most stable complex was docked with the binding energy of $-132.45 \mathrm{Kcal} / \mathrm{mol}$. The probable amino acid residues which are potentially involved in the interaction with the ligand have been depicted in fig. 12A. The docking studies for AaPCS revealed that the key residues viz., $\mathrm{Met}^{26}, \mathrm{Met}^{38}, \mathrm{Glu}^{39}, \mathrm{Phe}^{42}, \mathrm{Val}^{65}$ and $\mathrm{Leu}^{66}$ actively participated in the interaction with $\mathrm{CdCl}_{2}$ (Fig. 12B). In contrast, the residues involved in the interaction of anaPCS with $\mathrm{CdCl}_{2}$ were $\mathrm{Thr}^{108}$, Leu ${ }^{109}$, $\mathrm{Leu}^{112}, \mathrm{Ile}^{152}, \mathrm{Val}^{153}, \mathrm{Asn}^{154}, \mathrm{Ile}^{168}$ and Val ${ }^{219}$ (Fig. 12D). Key amino acid residues involved during sodium arsenate interaction with AaPCS were $\mathrm{Phe}^{84}$, Met ${ }^{88}$, Asp ${ }^{99}$, Lue $^{111}$ (Fig. 
12C). However, sodium arsenate and anaPCS interaction involved $\mathrm{Thr}^{108}, \mathrm{Leu}^{109}$, $\mathrm{Leu}^{112}, \mathrm{Ile}^{152}, \mathrm{Val}^{153}$, $\mathrm{Asn}^{154}, \mathrm{Ile}^{168}, \mathrm{Gly}^{217}, \mathrm{Val}^{2190}$ (Fig. 12E)

The result of molecular docking study revealed different amino acid residues were involved in the interaction of AaPCS with the ligand as those observed for anaPCS thus clearly portraying that members of PCS family specifically binds to ligand with varying amino acid residues that discloses a common interacting pattern.

\section{Protein-protein interaction}

The functional protein-protein interactive network of both AaPCS and anaPCS were predicted using STRING server at medium confidence level and the results were further validated by comparing proteinprotein interaction results by accessing PTIR database. At medium confidence level, AaPCS found to have strong interaction (Fig. 13A) with chloroplastic glutamatecysteine ligase (PGSC0003DMT400031827; score value 0.973 which regulates salicylic acid and phytoalexin biosynthesis thereby regulating tolerance response through GSH-dependent developmental pathway), chloroplastic glutathione synthetase (PGSC0003DMT400073627; score value 0.955 , which is exclusively involved in the glutathione biosynthesis and also regulates metal binding with in the plants) and lastly with gamma glutamyl transpeptidases (PGSC0003DMT400022803; score value 0.955 , which is mainly involve in the catabolism of glutathione, regulation of post-translational processing and cellular localization). On the other hand, anaPCS actively interacted (Fig. 13B) with ABC transporter ATP-binding protein (anaPCS; score value 0.999, which is involved in nutrients uptake including metals by utilizing energy from ATP binding), ABC transporter permease (anaPCS; score value 0.996, regulates metal transport) and Binding protein of $A B C$ transporter component (anaPCS; score value 0.995 , which are also involved in the import and export of various substrates including metal). From the results of the present study, it is evident that all the functional interactive partners of both AaPCS and anaPCS proteins belongs to transmembrane protein families exhibiting metal transport activity thus signifying the coordinated role of PCS proteins in the transportation of heavy metals. The complete list of the interacting partners from both AaPCS and anaPCS have been shown in supporting information (supplementary table 4 and 5).

\section{Superimposition results}

Several studies have reported that protein structures are conserved to a greater extent compared to sequences. Keeping these facts in minds, the predicted structures of both AaPCS and anaPCS were superimposed over each other to predict their topological properties (Supplementary fig. 7). The superimposition was performed using BLOSUM 62 matrix with gap penalty of 10 and extended penalty of 0.5. The superimposition results indicated that a total of 143 amino acid residues are substituted in between AaPCS and anaPCS with covering overall sequence similarity of $46.1 \%$ which was also further confirmed by the RMSD values of alpha carbon $\left(2.06 \mathrm{~A}^{\circ}\right)$ and back bone atoms $\left(2.07 \mathrm{~A}^{\circ}\right)$. In contrast, 
when AaPCS was allowed to be superimposed with the template 2BTW, the calculated RMSD values were $1.03 A^{\circ}$ for alpha carbon and $1.09 A^{\circ}$ for back bone atoms. Similarly, superimposition of anaPCS over template 2BTW showed RMSD values of $1.41 \mathrm{~A}^{\circ}$ both for alpha carbon and backbone atoms. The superimposition results confirmed the conservation of PCS functional domain structures as well as sequence across the divergent PCS members. However, structural alignment of functional domain of both AaPCS and anaPCS revealed some identical and non-identical (light blue) substitutions that extricated AaPCS domain from anaPCS. The amino acid substitutions were observed at position such as $\mathrm{Ser}^{5}$, Val $^{10}$, Pro $^{12}$, Glu ${ }^{34}$, Asn $^{47}$ and Gly ${ }^{75}$ in AaPCS when compared to anaPCS, where $\mathrm{Gln}^{5}$, $\mathrm{Thr}^{12}$, Asp ${ }^{241}$ and Phe ${ }^{242}$ were present.

\section{Gene ontology enrichment analysis}

The available protein sequences viz., AaPCS and anaPCS were subjected to CATH-Gene3D analysis to predict their subcellular localization and their functional annotation using gene ontology terms. Gene ontological (GO) terms serve as structural and functional descriptors characterizing genes and their products on the basis of their cellular, biological and molecular processes. The ReviGO analysis measured the biological function (supplementary fig. 8) of AaPCS protein revealed that $44 \%$ of the AaPCS protein is involved in the phytochelatin biosynthetic pathway (G0:0046938) and is potentially involved in the detoxification of metals such as arsenic, cadmium and copper (G0:0010273, G0:0046686, G0:0046685). The molecular function measured in terms of gene ontology revealed that the predicted proteins (56\%) is mostly engaged in glutathione gamma-glutamyl cysteinyl transferase activity (G0:0016756) and respond well under metal stress condition (GO:0005507, G0:0046870). On the other hand, CELLO2GO analysis (supplementary fig. 9 ) revealed that $56.8 \%$ of the proteins is localized into plasma membrane (score value 2.839 ) involved in phytochelatin biosynthetic process $(40.6 \%)$ and metal ion binding (35.7\%). For anaPCS, $17.8,11.1,4.4,8.9$ and $2.2 \%$ of proteins are involved in phytochelatin biosynthetic process, detoxification of copper, response to arsenic, detoxification of cadmium ion and arsenic transport respectively.

\section{Discussion}

In the present study, we aimed to enhance the heavy metal tolerance of $A$. annua hairy roots by expression of Anabaena PCC7120 phytochelatin synthase (anaPCS) gene. In their natural habitat, cyanobacteria including Anabaena are constantly exposed to adverse environmental condition such as high temperature, salinity, varied pH range and heavy metals (Rai et al. 2014, Singh et al. 2015) which make them an ideal system for extracting stress-tolerant genes. Cloning of anaPCS was achieved in $E$. coli (Chaurasia et al. 2008) and anaPCS overexpression in Anabaena had shown multiple stress tolerance in the cyanobacteria (Chaurasia et al. 2017). The present study shows firstever report of expression of cyanobacterial gene in $A$. annua. 
We began with three different tissue samples i.e., normal roots (derived from plantlets), hairy roots and transformed hairy roots (expressing anaPCS gene). Presence of rolB, presence of rolB and anaPCS and absence of both rolB and anaPCS confirmed the type of tissues as hairy roots, transformed hairy roots and normal roots respectively (Figs. 1 \& 4B).

Transformed hairy roots show significantly high amount of PCS expression owing to presence of two copies of PCS gene: one its own and another from Anabaena PCC 7120. As shown in Fig. 4 both As and Cd stress significantly induced the expression of anaPCS and AaPCS in transformed hairy roots and as a result As and Cd accumulation capacity was drastically enhanced in transformed hairy roots which accumulated 143.4 and $191 \%$ higher As and Cd than control. The value of bioconcentration factor (BCF) which gives an idea about capacity to accumulate heavy metals was significantly higher for transformed hairy roots. The BCF values of up to 21 and 9.7 for $C d$ and As respectively clearly explain that $\mathrm{Cd}$ is accumulated more than As however BCF value for both the heavy metals were significantly higher for transformed hairy roots as compared to non-transformed hairy roots or normal roots. In support of the present study, heterologous expression of NnPCS1 from lotus (with proved phytoremediation potential) conferred Cd tolerance in $A$. thaliana (Liu et al. 2012). Similarly, PCS gene from many other plants such as garlic and Indian mustard has been expressed in $A$. thaliana (Guo et al. 2008, Gasic and Korban 2007) and enhanced $C d$ and As tolerance significantly. In addition, heterologous expression of PCS gene from an aquatic macrophyte has provided better As and Cd tolerance in Tobacco (Shukla et al. 2012).

Both As and Cd are non-redox active metals however, causes significant oxidative damage to cell. Plant cells possess excellent defense system comprising of non-enzymatic and enzymatic antioxidant to mitigate their adverse effects.

Differential responses of four antioxidative enzymes were observed under As and Cd stress in all tissue samples. In transformed hairy roots, all antioxidative enzymes except catalase were drastically induced under heavy metal stresses. At different locations in the cell, $\mathrm{H}_{2} \mathrm{O}_{2}$ is converted to water and oxygen through APX, GPX and catalase. Only a slight increase in catalase activity after As and Cd exposure in all tissue samples (normal roots, hairy roots and transformed hairy roots), may probably be due to its high sensitivity towards superoxide radicals (Cakmak 2000). Catalase sensitivity under heavy metal stresses have also been reported in several plant species (Cao et al 2004; Gupta et al 2009) including A. annua (Rai et al 2011). Lesser catalase activity in heavy metal stressed transformed hairy roots was compensated by APX which possessed 31.9 and $34 \%$ greater activity compared to normal roots and hairy roots respectively. Transformed hairy roots possess better defense against heavy metal stress attributed to its capability to maintain high level of APX, GR and POD in unstressed and stressed conditions. High activity of GR has probably contributed towards better As and $\mathrm{Cd}$ tolerance in transformed hairy roots of $A$. annua because GR plays a significant role in heavy metal stresses by maintaining high GSH:GSSG ratio in the cell via recycling GSSG to GSH. As- and Cd-stress induced antioxidative enzyme activities including that for APX, GR and POD in several plants (Kumar et al 2014, Rai et al 2011, Zhang et al 2007). 
In addition to antioxidative enzymes, low molecular weight thiols also play critical role in maintaining the overall oxidative balance in the cell. Their maximum induction in transformed hairy roots (with anaPCS gene) under As- and Cd-stresses (Fig. 5A) is indicative of better stress tolerance. GSH is one of the low molecular weight thiols with at least two crucial functions: synthesis of PCs and maintaining the ascorbate-glutathione cycle, both as part of antioxidative defense (Cobbett 2000). In the present study, transformed hairy roots expressing anaPCS showed approximately 4-folds and 2-folds higher PCs level as compared to normal roots and non-transformed roots. As PCs are known to form metal-complexes which are sequestered into the vacuoles, boosted PCs level in transformed hairy roots suggests their potential to efficiently detoxify As and $\mathrm{Cd}$. This can also be correlated with higher accumulation of As and Cd in transformed hairy roots than other samples and high GR activity which generates enough GSH to be used for PCs synthesis. Overexpression of AaPCS and anaPCS in transformed hairy roots supports the higher PCs level. However, in the present study, GSH levels were dropped under As and Cd stress. As product of PCS gene is being synthesized in high amount, lower GSH level in transformed hairy roots was probably because of the overconsumption for PCs synthesis and not due to its inhibition. The result finds support with Samane et al. (2007), Ben Ammar et al. (2008) and Meng et al. (2019) who have found lower GSH but higher PCs level in heavy metal stressed Arabidopsis thaliana, tomato and lettuce respectively.

In-silico analysis gave several confirmatory pieces of evidences for the proper functioning of a cyanobacterial anaPCS in a higher plant $A$. annua. The presence of conserved catalytic domain (Fig. 9) as well as functional domain structures (Supplementary Fig. 7) among anaPCS and AaPCS may have led cyanobacterial anaPCS to function properly in $A$. annua hairy roots. Protein-protein interaction analysis confirmed anaPCS involvement in heavy metal transport as their functional interacting partners belong to transmembrane protein families having metal transport activity. This further get confirmation through gene ontology analysis where proportions of anaPCS proteins have been shown to be involved in biological processes like phytochelatin biosynthesis, arsenic transport and detoxification of cadmium ions.

In addition to better tolerance towards As and Cd, transformed hairy roots also showed the significantly greater synthesis of its popular antimalarial compound artemisinin (Fig. 7). Artemisnin biosynthesis have been shown to be improved under both arsenic (Kumari et al. 2018) and cadmium stress (Li et al. 2012). Owing to its immense therapeutic potential as antimalarial, anticarcinogenic, antimicrobial and many others, its global demand is always high and needs to be supplied enough in order to meet high demand (Pandey and Pandey-Rai 2016). Recently artemisinin has been suggested to be repurposed for the treatment of COVID-19 and other related respiratory disorders which will further increase its demand (Cheong et al. 2020). Higher artemisinin biosynthesis with enhanced heavy metal tolerance in hairy roots of $A$. annua expressing anaPCS gene, can serve a dual purpose.

\section{Conclusion}


Heterologous expression of a cyanobacterial phytochelatin synthase (anaPCS) in hairy roots of $A$. annua was successfully achieved which led to enhanced heavy metal (arsenic and cadmium) tolerance. Under conditions of heavy metal stresses, greater accumulation of arsenic and cadmium, higher biosynthesis of phytochelatins, and activation of enzymatic and non-enzymatic antioxidants have made transformed hairy roots (expressing anaPCS) more tolerant to arsenic and cadmium stresses. Due to conserved catalytic domain and functional domain among anaPCS and AaPCS, the former could have performed in a different system (higher plant). In-silico analysis revealed involvement of anaPCS protein in heavy metal transport, phytochelatin biosynthesis and detoxification of cadmium and arsenic. Enhanced artemisinin biosynthesis in transformed hairy roots further gave scope of their probable use in phytoremediation and extraction of artemisinin from used hairy roots.

\section{Declarations}

\section{Acknowledgement}

The authors acknowledge Department of Science and Technology (DST- SERB), Govt. of India, for financial support (EMR/2017/001343). They also acknowledge the facility provided by Department of Biotechnology funded Sub-Distributed Information Centre, Centre for Bioinformatics, School of Biotechnology, Banaras Hindu University. DST-PURSE, FIST and Central Instrument facility, Department of Botany, Banaras Hindu University is also acknowledged for providing financial and instrument support during the experiments. Authors also sincerely acknowledge Prof. L C Rai, Department of Botany, Institute of Science, Banaras Hindu University, Varanasi, for his support.

Authors' contribution: NP, KKR, SKR and SPR designed the experiments. NP and KKR conducted the experiments. NP, KKR, SKR and SPR analyzed the data and wrote the manuscript.

Funding: Department of Science and Technology (DST- SERB), Govt. of India, (EMR/2017/001343).

Conflict of interest: Authors declare no conflict of interest.

Availability of data and material: All raw data has been provided as supplementary material.

\section{References}

Agostini E, Talano MA, González PS,Oller ALW, Medina MI (2013) Application of hairy roots for phytoremediation: what makes them an interesting tool for this purpose?Appl Microbiol Biotechnol 97(3):1017-1030.

Anderson ME (1985) Determination of glutathione and glutathione disulfide in biological samples. Methods Enzymol113:548-555.

Bailey TL, Williams N, MislehC, Li WW (2006) MEME: discovering and analyzing DNA and protein sequence motifs. Nucleic Acids Res 34:369-373. 
Ben Ammar W, Mediouni C, Tray B, Ghorbel MH, Jemal F (2008) Glutathione and phytochelatin contents in tomato plants exposed to cadmium. Biol Plant 52:314-320. https://doi.org/10.1007/s10535-0080065-9.

Benkert P, Kunzli M, Schwede T (2009) QMEAN server for protein model quality estimation. Nucleic Acids Res 37:W510-W514.

Berman HM, Westbrook J, Feng Z, Gilliland G, Bhat TN, Weissig H et al (2000) The protein data bank. Nucleic Acids Res 28: 235-242.

Bleeker PM, Hakvoort HW, Bliek M, Souer E, Schat H (2006) Enhanced arsenate reduction by a CDC25-like tyrosine phosphatase explains increased phytochelatin accumulation in arsenate-tolerant Holcus lanatus. The Plant J45 (6):917-929.

Cakmak I (2000) Tansley Review No. 111 Possible roles of zinc in protecting plant cells from damage by reactive oxygen species. New Phytol 146(2):185-205.

Cao X, Ma LQ, Tu C (2004) Antioxidant responses to arsenic in the arsenic hyperaccumulator Chinese brake fern (Pteris vittata L.). Environ Pollut 128:317-325. doi: 10.1016/j.envpol.2003.09.018

Castrignano T, De Meo PD, Cozzetto D, Talamo IG, Tramontano A (2006) The PMDB protein model database. Nucleic Acids Res. 34:306-309.

Chaurasia N, Mishra Y Rai LC (2008) Cloning expression and analysis of phytochelatin synthase (pcs) gene from Anabaena sp. PCC 7120 offering multiple stress tolerance in Escherichia coli. Biochem Biophys Res Commun 376(1):.225-230.

Chaurasia N, Mishra Y, Chatterjee A, Rai R., Yadav S. Rai LC (2017) Overexpression of phytochelatin synthase (pcs) enhances abiotic stress tolerance by altering the proteome of transformed Anabaena sp. PCC 7120. Protoplasma 254(4):1715-1724.

Cheong DH, Tan WD, Wong WF Tran T (2020) Anti-malarial drug, artemisinin and its derivatives for the treatment of respiratory diseases. Pharmacol Res 104901.

Cobbett CS (2000) Phytochelatins and their roles in heavy metal detoxification. Plant physiol 123(3):825832.

Colovos C, Yeates TO (1993) ERRAT: an empirical atom-based method for validating protein structures. Protein Sci 2: 1511-1519

de Castro E., Sigrist C. J. A, Gattiker A, Bulliard V, Langendijk-Genevaux P S, Gasteiger E, et al. (2006). ScanProsite: detection of PROSITE signature matches and ProRule-associated functional and structural residues in proteins. Nucleic Acids Res 1: 362-365. 
Gasic K, Korban SS (2007) Transgenic Indian mustard (Brassica juncea) plants expressing an Arabidopsis phytochelatin synthase (AtPCS1) exhibit enhanced As and Cd tolerance. Plant Mol Boil 64(4)::361-369.

Guo J, Dai X, Xu W and Ma M (2008) Overexpressing GSH1 and AsPCS1 simultaneously increases the tolerance and accumulation of cadmium and arsenic in Arabidopsis thaliana. Chemosphere 72(7):10201026.

Gupta M, Sharma P, Sarin NB, Sinha AK (2009) Differential response of arsenic stress in two varieties of Brassica juncea L. Chemosphere 74:1201-1208. doi:10.1016/j.chemosphere. 2008.11.023

Gutierrez-Valdes N, Häkkinen ST, Lemasson C, Guillet M, Oksman-Caldentey KM, Ritala A, Cardon F (2020) Hairy Root Cultures-A Versatile Tool with Multiple Applications. Front Plant Sci 11:33. doi: $10.3389 /$ fpls.2020.00033

Hall TA (1999) BioEdit: a user-friendly biological sequence alignment editor and analysis program for Windows 95/98/NT. In Nucleic acids symposium series [London]: Information Retrieval Ltd., c1979c2000. 41(41): 95-98

Hooykaas PJJ, Klapwijk PM, Nuti MP, Schilperoort RA and Rörsch A (1977) Transfer of the Agrobacterium tumefaciens Ti plasmid to avirulent agrobacteria and to Rhizobium ex planta. Microbiology 98(2):477484

Huang B (2009). MetaPocket: a meta approach to improve protein ligand binding site prediction. OMICS 13(4): 325-330.

Ismael MA, Elyamine AM, Moussa MG, Cai M, Zhao X, Hu C (2019) Cadmium in plants: uptake, toxicity, and its interactions with selenium fertilizers. Metallomics 11(2): 255-277.

Jones P, Binns D, Chang HY, Fraser M., Li W, McAnulla C., et al. (2014) InterProScan 5: genome-scale protein function classification. Bioinformatics 30: 1236-1240

Khalid S, Shahid M, Niazi NK, Rafiq M, Bakhat HF, Imran M, Abbas T, Bibi I, Dumat C (2017) Arsenic behaviour in soil-plant system: Biogeochemical reactions and chemical speciation influences. In Enhancing Cleanup of Environmental Pollutants (pp. 97-140). Springer, Cham.

Krzywinski M, Schein J, Birol I, Connors J, Gascoyne R, Horsman D et al. (2009) Circos: an information aesthetic for comparative genomics. Genome Res 19: 1639-1645.

Kumar A, Singh RP, Singh PK, Awasthi S, Chakrabarty D, Trivedi PK, Tripathi RD (2014) Selenium ameliorates arsenic induced oxidative stress through modulation of antioxidant enzymes and thiols in rice (Oryza sativa L.). Ecotoxicology 23(7):1153-63. doi: 10.1007/s10646-014-1257-z. 
Kumari A, Pandey N, Pandey-Rai S (2017) Protection of Artemisia annua roots and leaves against oxidative stress induced by arsenic. Biologia Plantarum 61(2):367-377.

Kumari A, Pandey N Pandey-Rai S (2018) Exogenous salicylic acid-mediated modulation of arsenic stress tolerance with enhanced accumulation of secondary metabolites and improved size of glandular trichomes in Artemisia annua L. Protoplasma 255(1): 139-152.

Laskowski RA, Chistyakov VV, Thornton JM (2005) PDBsum more: new summaries and analyses of the known 3D structures of proteins and nucleic acids. Nucleic Acids Res. 1: D266-D268.

LeBlanc MS, McKinney EC, Meagher RB, Smith AP (2013) Hijacking membrane transporters for arsenic phytoextraction. J Biotechnol 163(1):1-9.

Li X, Zhao M, Guo L, Huang L (2012) Effect of cadmium on photosynthetic pigments, lipid peroxidation, antioxidants, and artemisinin in hydroponically grown Artemisia annua. J Environ Sci 24(8):1511-1518.

Liu Z, Gu C, Chen F, Yang D, Wu K, Chen S, Jiang J, Zhang Z (2012) Heterologous expression of a Nelumbo nucifera phytochelatin synthase gene enhances cadmium tolerance in Arabidopsis thaliana. Appl Biochem Biotech 166(3):722-734.

Lovell SC, Davis IW, Arendall WB, de Bakker PI, Word JM, Prisant MG, et al. (2003) Structure validation by Ca geometry: phi-psi and C-beta deviation. Proteins 50, 437-450.

Macindoe G, Mavridis L, Venkatraman V, Devignes M, Ritchie D (2010) Hex Server: an FFT-based protein docking server powered by graphics processors. Nucleic Acids Res 38: W445-W449.

Maiti R, Van Domeselaar GH, Zhang H, Wishart DS (2004) SuperPose: a simple server for sophisticated structural superposition. Nucleic Acids Res. 32: W590-W594.

Marchler-Bauer A, Lu S, Anderson JB, Chitsaz F, Derbyshire MK, DeWeese-Scott C et al. (2011) CDD: a Conserved Domain Database for the functional annotation of proteins. Nucleic Acids Res 39: D225D229.

Meng Y, Zhang L, Wang L, Zhou C, Shangguan Y, Yang Y (2019) Antioxidative enzymes activity and thiol metabolism in three leafy vegetables under Cd stress. Ecotoxicol Environ Saf 173:214-224.

Mukhopadhyay R, Bhattacharjee H, Rosen BP (2014) Aquaglyceroporins: generalized metalloid channels. Biochim Biophys Acta 1840(5):1583-1591.

Nagalakshmi N, Prasad MNV (2001) Responses of glutathione cycle enzymes and glutathione metabolism to copper stress in Scenedesmus bijugatus. Plant Sci, 160(2):291-299.

Pandey N, Pandey-Rai S (2014) Short term UV-B radiation-mediated transcriptional responses and altered secondary metabolism of in vitro propagated plantlets of Artemisia annua L. Plant Cell Tissue Organ 
Cult 116(3):371-385.

Pandey N, Pandey-Rai S (2016) Updates on artemisinin: an insight to mode of actions and strategies for enhanced global production. Protoplasma 253(1): 15-30.

Patra N, Srivastava AK (2014) Enhanced production of artemisinin by hairy root cultivation of Artemisia annua in a modified stirred tank reactor. Appl Biochem Biotech 174(6):2209-2222.

Pu GB, Ma DM, Chen JL, Ma LQ, Wang H, Li GF, Ye HC, Liu BY (2009) Salicylic acid activates artemisinin biosynthesis in Artemisia annua L. Plant Cell Rep, 28(7):1127-1135.

Rai R, Pandey S, Rai SP (2011) Arsenic-induced changes in morphological, physiological, and biochemical attributes and artemisinin biosynthesis in Artemisia annua, an antimalarial plant. Ecotoxicology 20(8):1900-1913.

Rai R, Pandey S, Shrivastava AK, Pandey-Rai S (2014) Enhanced photosynthesis and carbon metabolism favor arsenic tolerance in Artemisia annua, a medicinal plant as revealed by homology-based proteomics. Int J Proteomics 2014:163962

Rippka R, Deruelles J, Waterbury JB, Herdman M, Stanier RY (1979) Generic assignments, strain histories and properties of pure cultures of cyanobacteria. Microbiology, 111(1):1-61.

Sambrook J, Russell DW (2001) Molecular Cloning. A laboratory manual., ed. CS Harbor. Vol. 2, 3rd edn. Cold Spring Harbor Laboratory Press, New York.

Semane B, Cuypers A, Smeets K, Van Belleghem F, Horemans N, Schat H, Vangronsveld J (2007) Cadmium responses in Arabidopsis thaliana: glutathione metabolism and antioxidative defence system. Physiol Plant 129: 519-528. https:// doi.org/10.1111/j.1399-3054.2006.00822.x

Shahi SK, Singh VK, Kumar A, Gupta SK, Singh SK (2013) Interaction of dihydrofolate reductase and aminoglycoside adenyl transferase enzyme from Klebsiella pneumoniae multidrug resistant strain DF12SA with clindamycin: a molecular modeling and docking study. J Mol Model 19: 973-983.

Shukla D, Kesari R, Mishra S, Dwivedi S, Tripathi RD, Nath P, Trivedi PK (2012) Expression of phytochelatin synthase from aquatic macrophyte Ceratophyllum demersum $L$. enhances cadmium and arsenic accumulation in tobacco. Plant cell rep 31(9):1687-1699.

Sillitoe I, Lewis TE, Cuff A, Das S, Ashford P, Dawson NL et al (2015) CATH: comprehensive structural and functional annotations for genome sequences. Nucleic Acid Res. 43: D376-D381.

Srivastava AK, Ara A, Bhargava P, Mishra Y, Rai SP, Rai LC (2007) A rapid and cost-effective method of genomic DNA isolation from cyanobacterial culture, mat and soil suitable for genomic fingerprinting and community analysis. J Appl Phycol 19(4):.373-382. 
Supek F, Bošnjak M, Škunca N, Šmuc T (2011) REVIGO summarizes and visualizes long lists of gene ontology terms. PloS One 6(7): e21800.

Szklarczyk D, Franceschini A, Wyder S, Forslund K, Heller D, HuertaCepas J et al. (2015) STRING v10: protein-protein interaction networks, integrated over the tree of life. Nucleic Acids Res. 43: D447-D452.

Tamura K, Stecher G, Peterson D, Filipski A, Kumar S (2013) MEGA6: molecular evolutionary genetics analysis version 6.0. Mol. Biol. Evol. 30: 2725-2729.

Usman ARA, Alkredaa RS, Al-Wabel MI (2013) Heavy metal contamination in sediments and mangroves from the coast of Red Sea: Avicennia sp. Marina as potential metal bioaccumulator. Ecotoxicol. Environ. Saf. 97: 263-270. https://doi.org/10. 1016/j.ecoenv.2013.08.009

Vatamaniuk OK, Mari S, Lu YP, Rea PA (1999) AtPCS1, a phytochelatin synthase from Arabidopsis: isolation and in vitro reconstitution. Proc Natl Acad Sci U S A 96(12):7110-7115.

Wiederstein M, Sippl MJ (2007) ProSA-web: interactive web service for the recognition of errors in threedimensional structures of proteins. Nucleic Acids Res. 35: W407-W410.

Willard L, Ranjan A, Zhang H, Monzavi H, Boyko RF, Sykes BD, Wishart DS (2003) VADAR: a web server for quantitative evaluation of protein structure quality. Nucleic Acids Res 31(13): 3316-3319.

Xu D, Zhang Y (2011) Improving the physical realism and structural accuracy of protein models by a twostep atomic-level energy minimization. Biophys J 101(10): 2525-2534.

Zhang FQ, Wang YS, Lou ZP, Dong JD (2007) Effect of heavy metal stress on antioxidative enzymes and lipid peroxidation in leaves and roots of two mangrove plant seedlings (Kandelia candel and Bruguiera gymnorrhiza). Chemosphere 67(1):44-50. doi: 10.1016/j. chemosphere.2006.10.007.

Zhang W, Lin K, Zhou J, Zhang W, Liu L, Han X (2013) Spatial distribution and toxicity of cadmium in the joint presence of sulfur in rice seedling. Environ Toxicol Pharmaco/36(3):1235-1241.

Zhao SS, Zeng MY (1985) Spectrometric high-pressure liquid chromatographical (hplc) studies on the analysis of qinghaosu. Planta Med 51:233-23

\section{Figures}




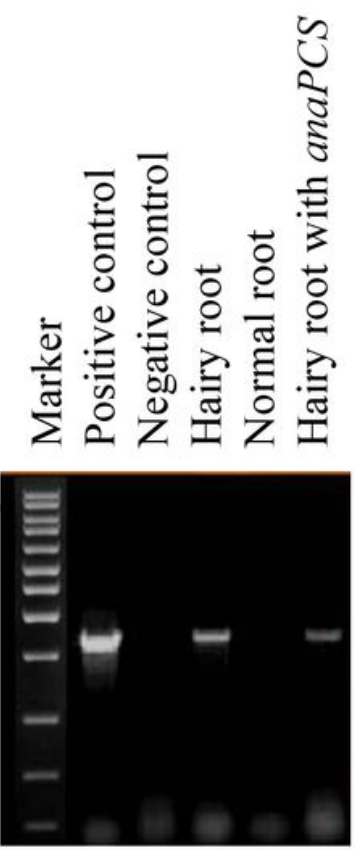

Figure 1

RT-PCR for expression analysis of rolB gene in hairy root, normal root and transformed hairy roots expressing anaPCS gene.
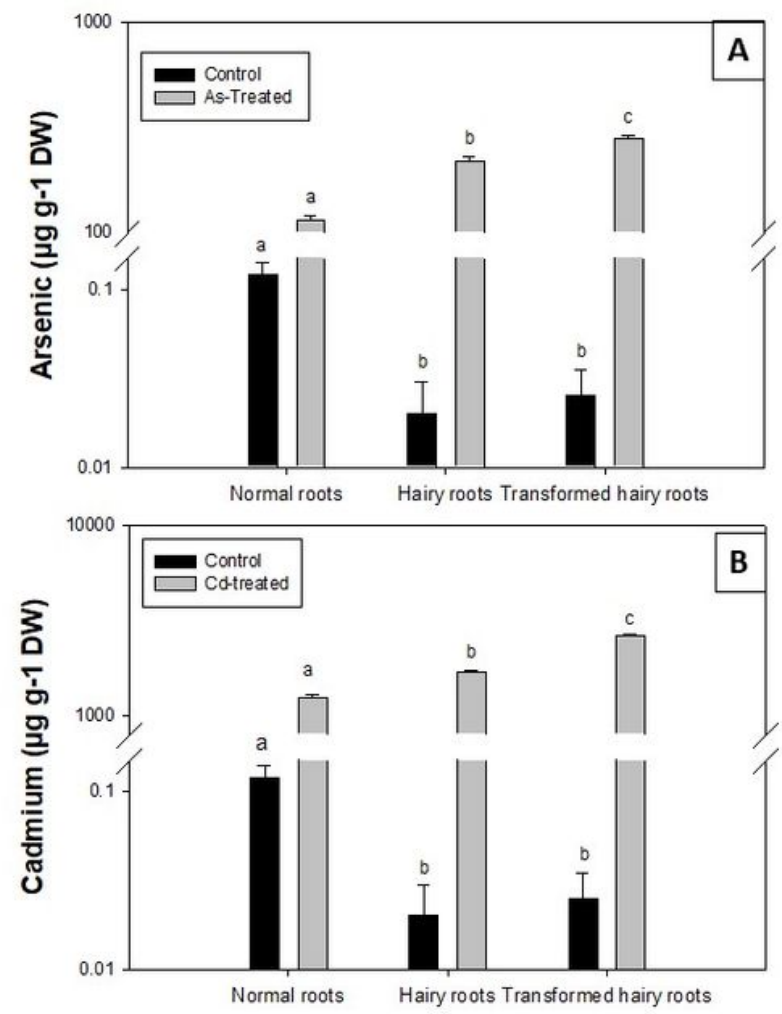

Page 25/35 
Figure 2

Heavy metal accumulation in normal roots, hairy roots and transformed hairy roots (expressing anaPCS gene). (A) Arsenic content (B) Cadmium content. Vertical bars denote mean \pm SD and different alphabets show statistical significance among treatments (control / As-treatment / Cd-treatment) at $p=<0.05$.

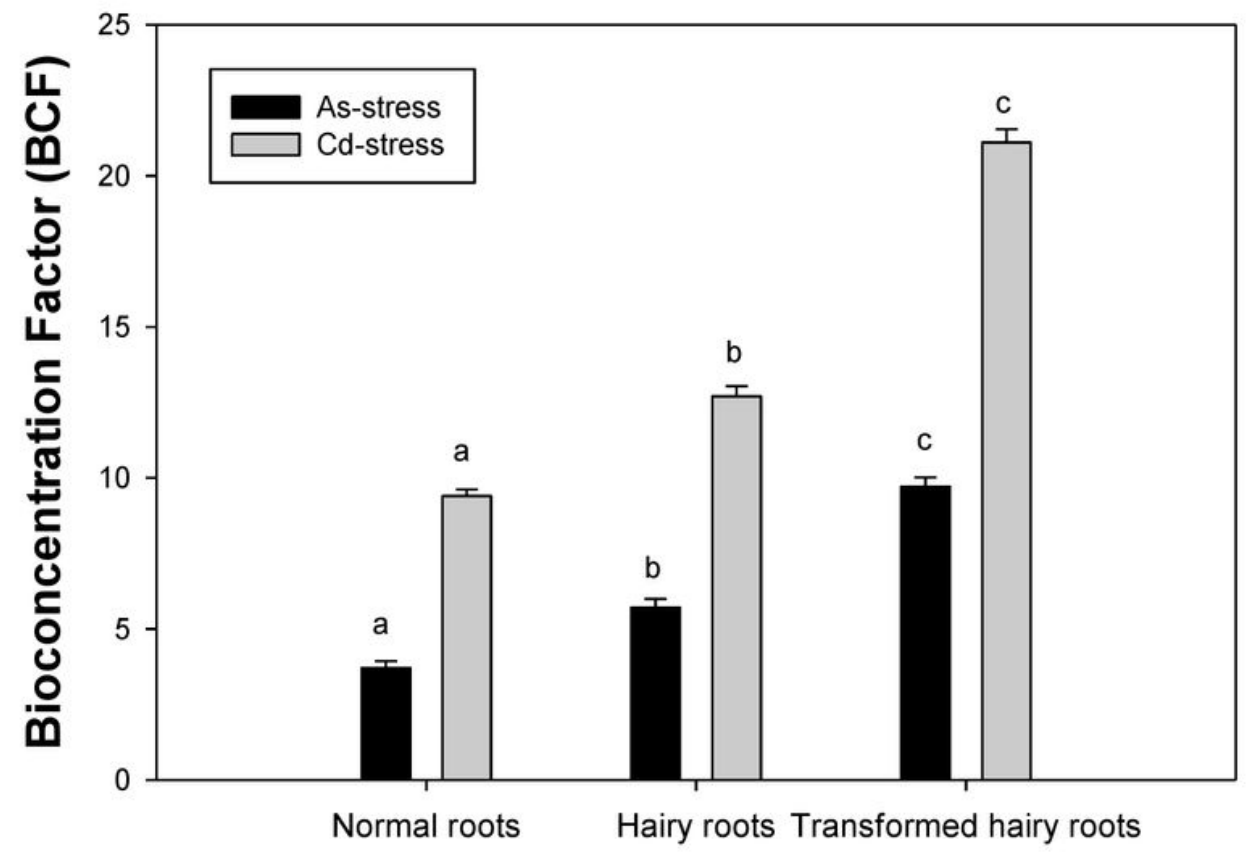

Figure 3

Bioconcentration factor (BCF) of arsenic and cadmium-stressed normal roots, hairy roots and transformed hairy roots (expressing anaPCS gene). Vertical bars denote mean \pm SD and different alphabets show statistical significance among treatments (control / As-treatment / Cd-treatment) at $p=$ $<0.05$. 


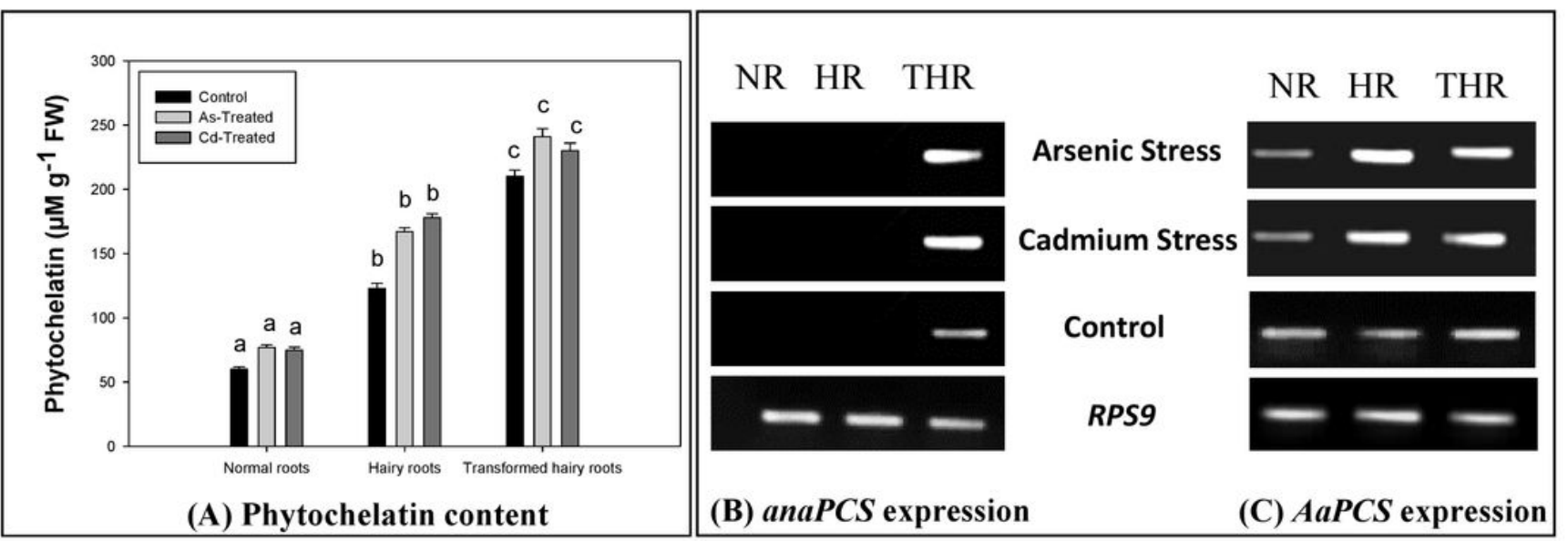

Figure 4

Phytochelatin content and phytochelatin synthase expression in normal roots, hairy roots and transformed hairy roots (expressing anaPCS gene) under arsenic and cadmium stress. (A) Phytochelatin content (B) RT-PCR for expression analysis of Anabaena PCC 7120 PCS gene (anaPCS) (C) RT-PCR for expression analysis of Artemisia annua PCS (AaPCS). 


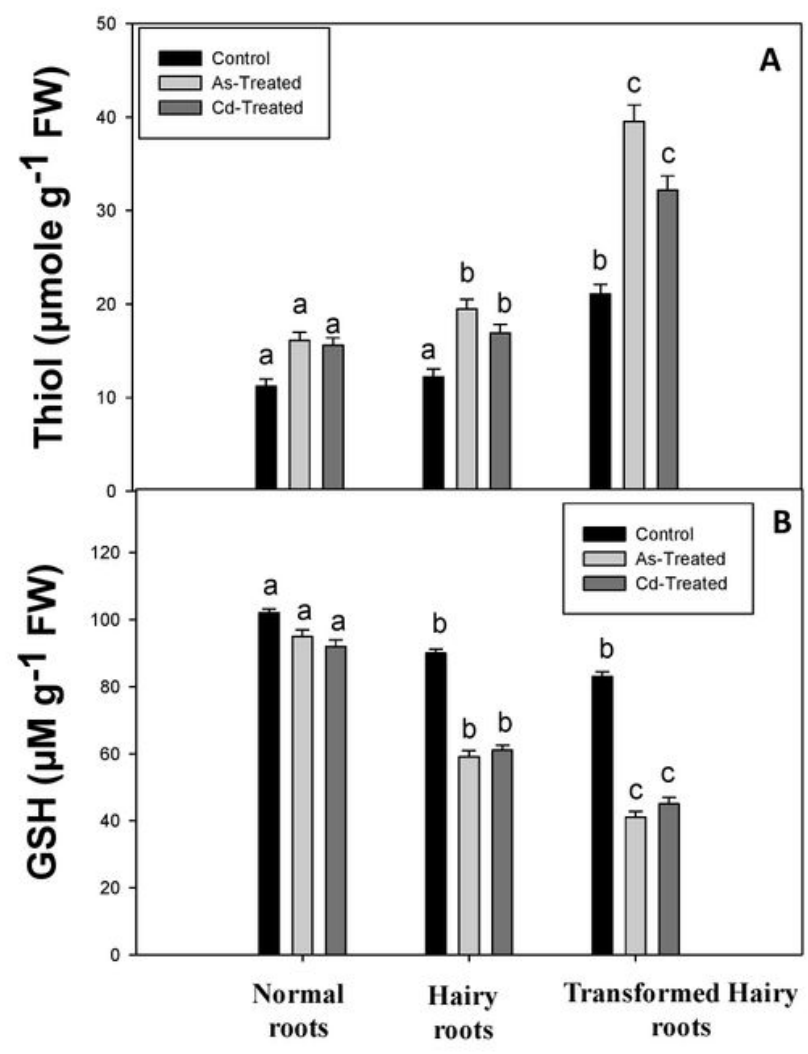

Figure 5

(A) Thiol content and (B) GSH content in normal roots, hairy roots and transformed hairy roots (expressing anaPCS gene) under arsenic and cadmium stress. Vertical bars denote mean \pm SD and different alphabets show statistical significance among treatments (control / As-treatment / Cdtreatment) at $p=<0.05$. 


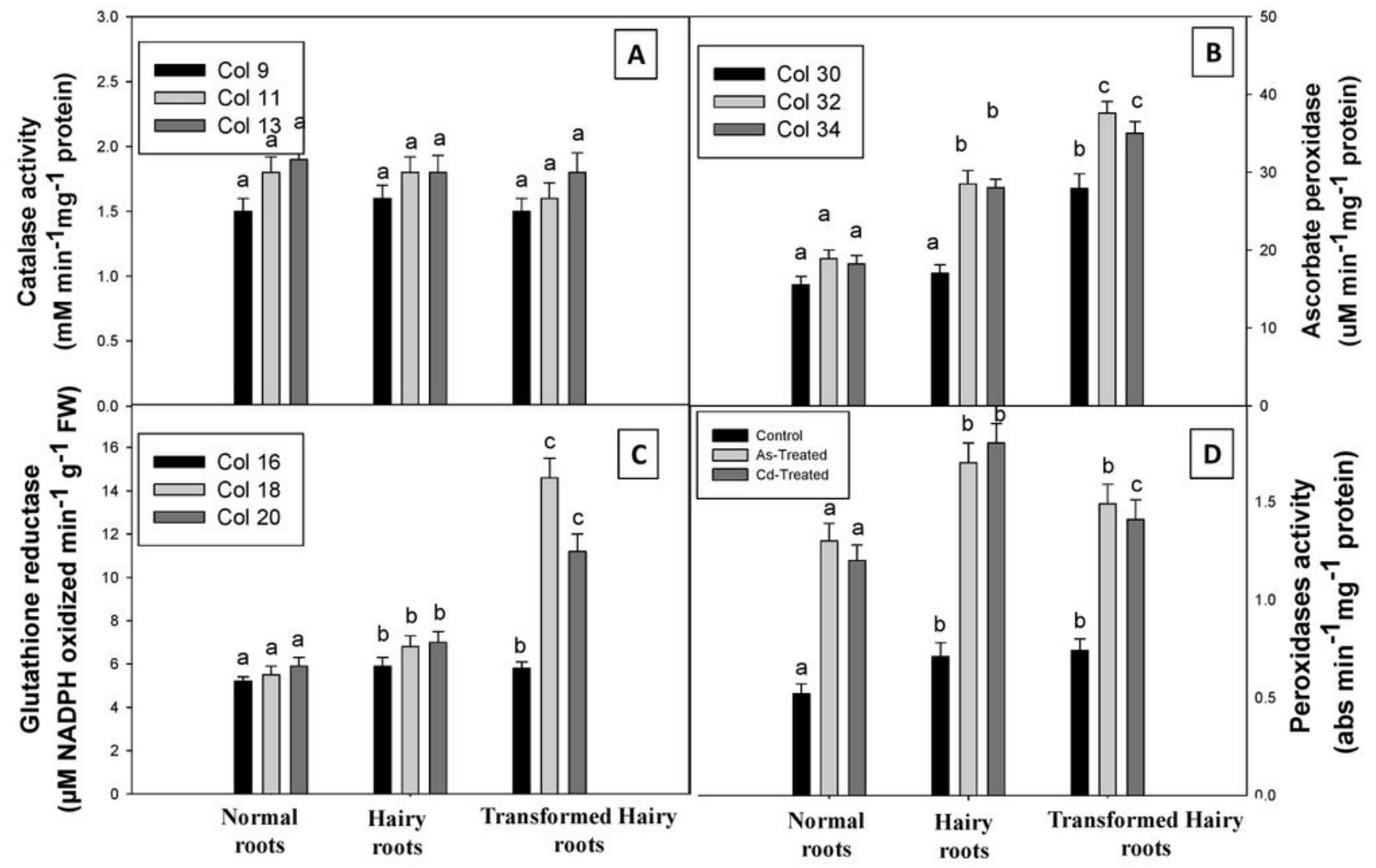

Figure 6

Antioxidative enzyme activities of (A) Catalase (B) Ascorbate peroxidase (C) Glutathione reductase (D) Peroxidases, in normal roots, hairy roots and transformed hairy roots (expressing anaPCS gene) under arsenic and cadmium stress. Vertical bars denote mean \pm SD and different alphabets show statistical significance among treatments (control / As-treatment / Cd-treatment) at $p=<0.05$. 


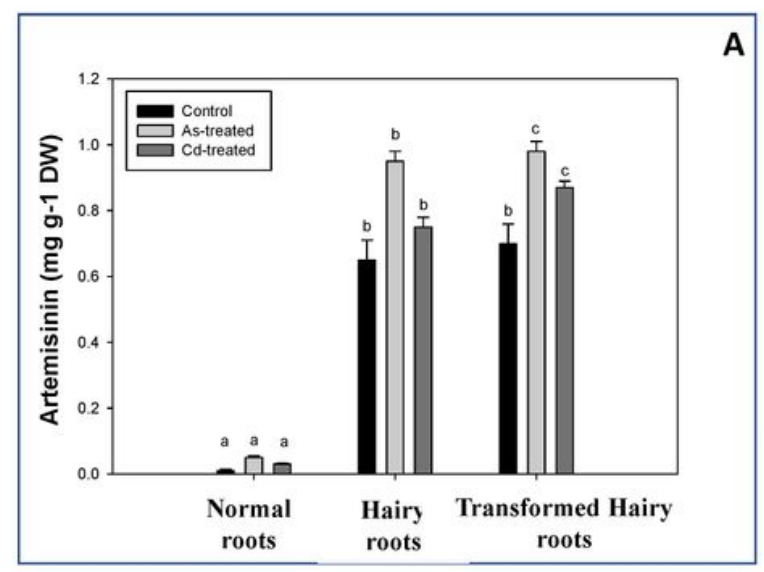

\begin{tabular}{|c|c|c|c|}
\hline $\mathrm{C} \quad \mathrm{Cd}$ As & C Cd As & C $\quad \mathrm{Cd}$ As & B \\
\hline$-\infty$ & & 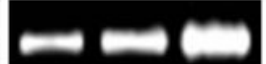 & $A D S$ \\
\hline$\ldots-\infty$ & & - & CYPAIVI \\
\hline 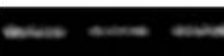 & $=$ & - & DBR2 \\
\hline$-m-$ & 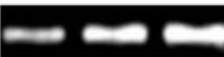 & & $A L D H I$ \\
\hline 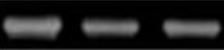 & $m e$ & $=-$ & RPS 9 \\
\hline Normal root & Hairy root & $\begin{array}{l}\text { Transformed Hairy } \\
\text { roots }\end{array}$ & \\
\hline
\end{tabular}

Figure 7

(A) Artemisinin content (B) expression analysis of artemisinin biosynthetic enzymes (ADS, CYP71AV1, DBR2 and ALDH1) through RT-PCR, in normal roots, hairy roots and transformed hairy roots (expressing anaPCS gene) under arsenic and cadmium stress. 
(A)

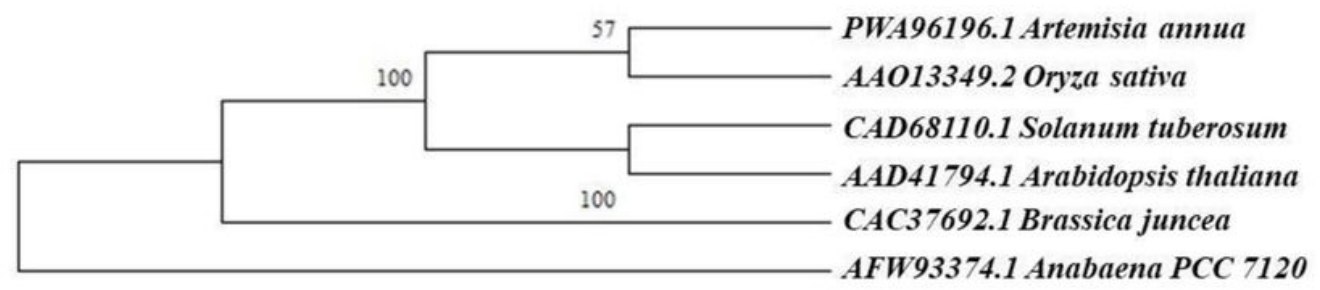

(B)
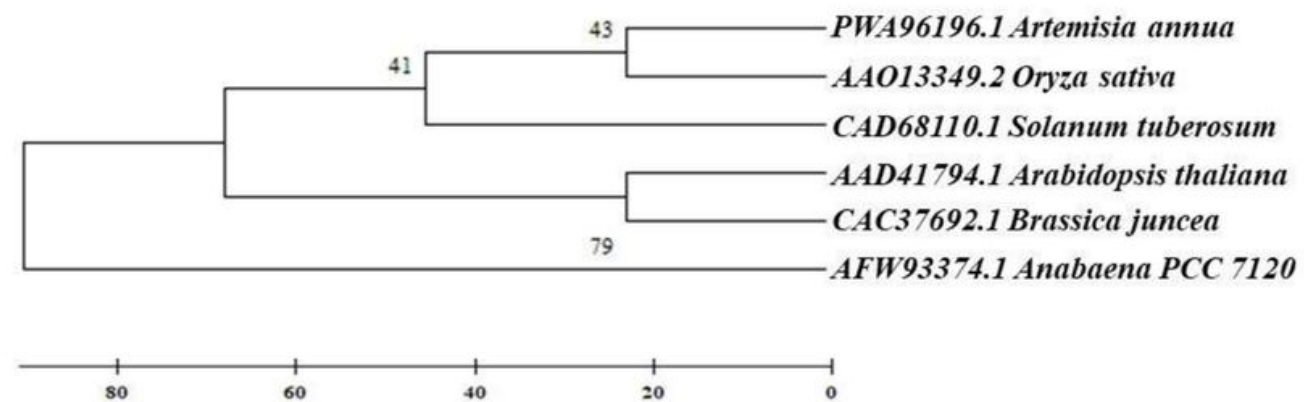

Figure 8

Phylogenetic tree (A) UPGMA method (B) Maximum Parsimony method showing origin, evolutionary relationship and functional homology of phytochelatin synthase protein in A.annua, S.tuberosum, A.thaliana, B.juncea, O.sativa and Anabaena PCC 7120. The trees were generated by using 1000 boot replication values based on maximum likelihood methods. 


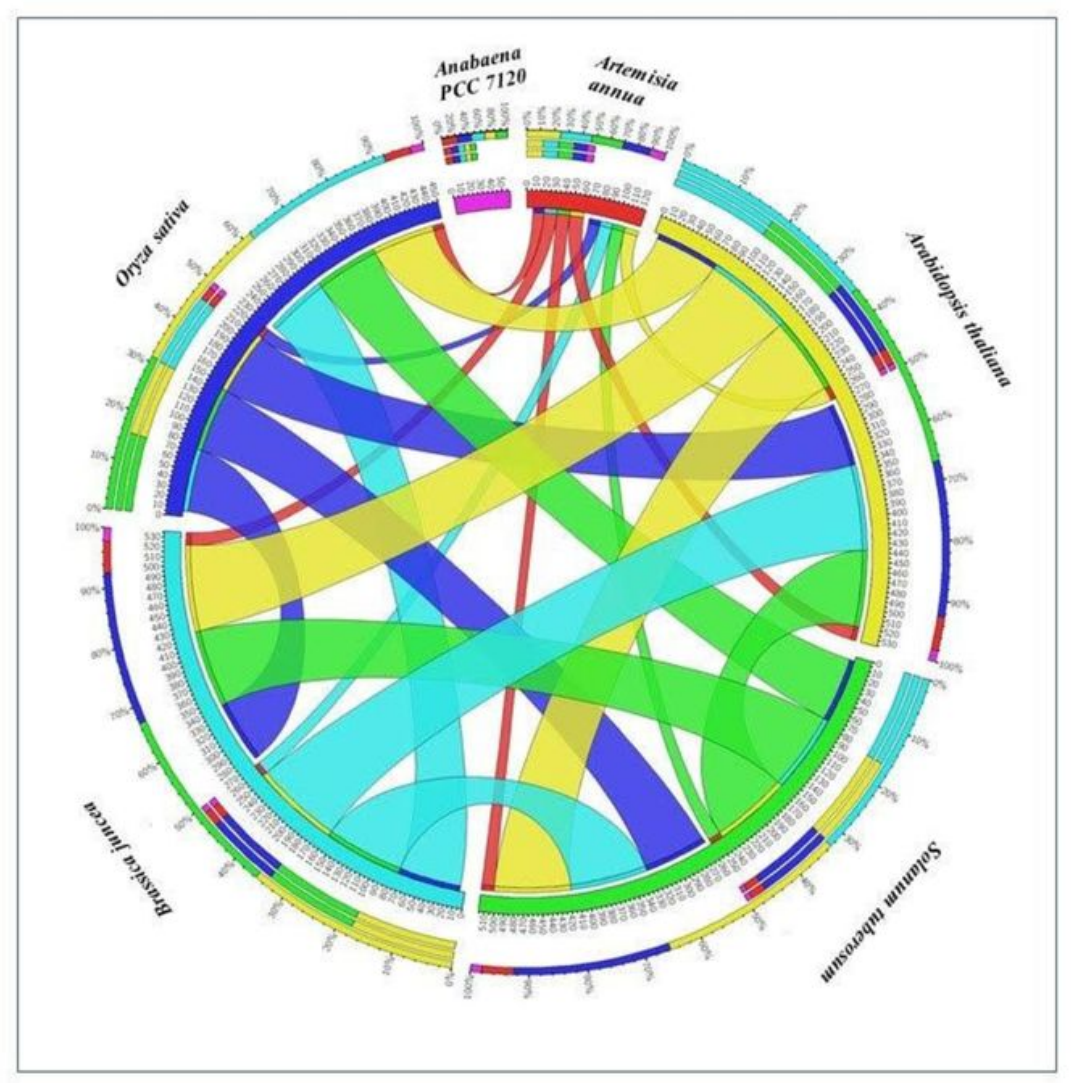

Figure 9

Comparative analysis of similarities and differences in phytochelatin synthase (PCS) protein among A. annua, S. tuberosum, A. thaliana, B. juncea, O. sativa and Anabaena PCC 7120. The Circos software was used to generate circular map based on similarity percentage matrices using Clustal W algorithm. 


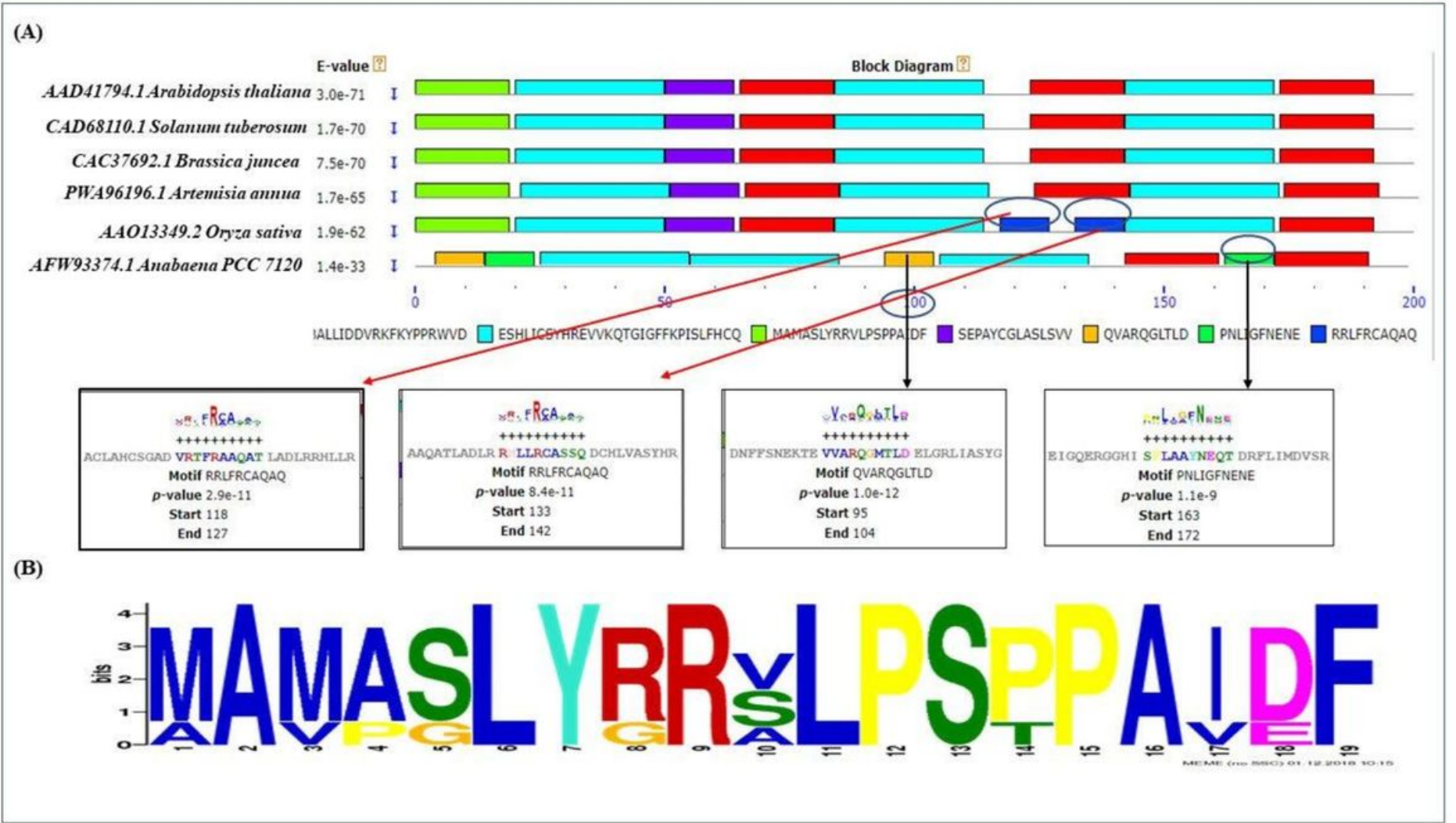

Figure 10

The motif scan analysis showing distribution and presence /absence of common and uncommon motifs found in A. annua, S. tuberosum, A. thaliana, B. juncea, O. sativa and Anabaena PCC 7120 discovered through MEME and MAST analysis. (A) The block diagram showing the sequence of discovered motifs for AaPCS. The red arrows indicate the presence of uncommon motif in 0. sativa and black arrows indicate the presence of uncommon motifs in Anabaena PCC 7120 which are absent in other members. (B) The sequential logo of the motif 1 showing consensus sequences present in all the representatives' members. 


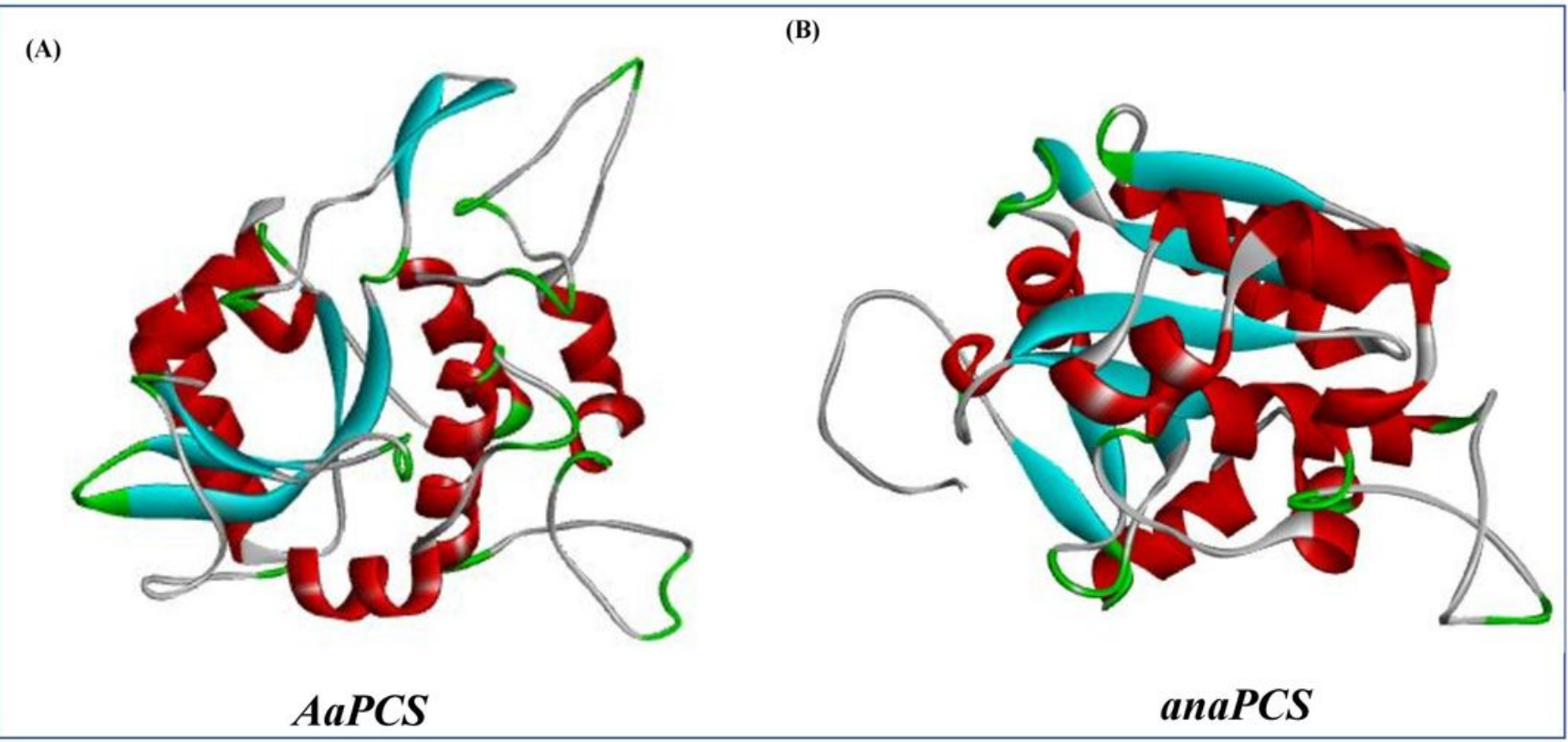

Figure 11

Predicted structures of functional domain of (A) A. annua PCS (AaPCS) (B) Anabaena PCC 7120 PCS (anaPCS) generated and visualized through MODELLER module of Discovery Studio 3.0.

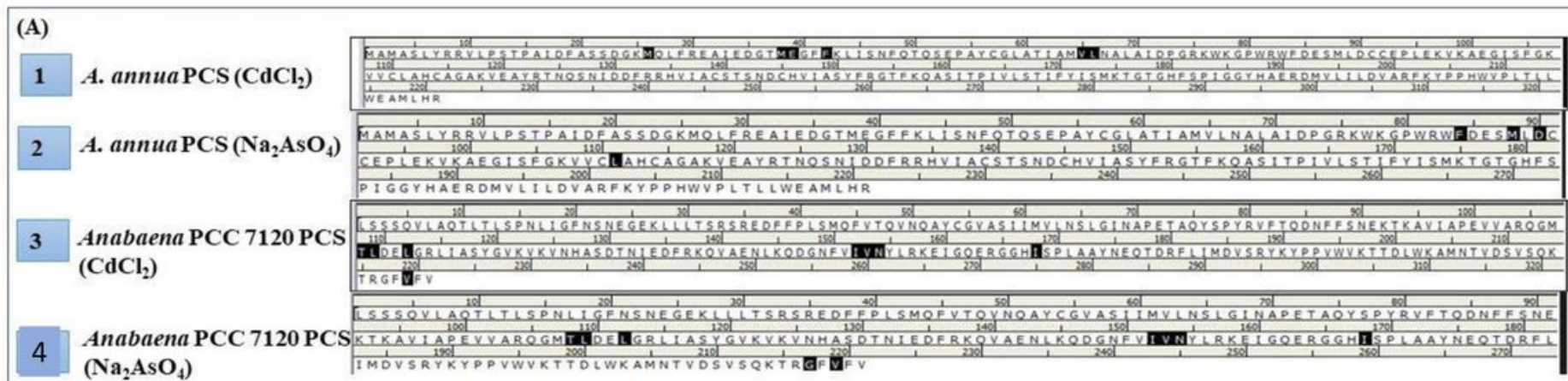

(B)

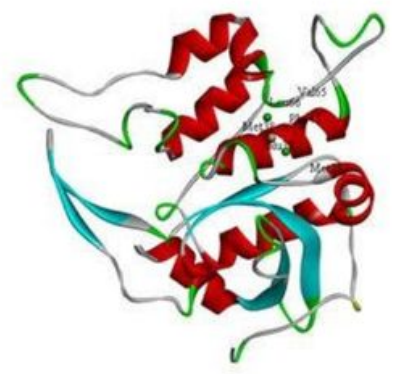

A. annua $\mathrm{PCS}\left(\mathrm{CdCl}_{2}\right)$
(C)

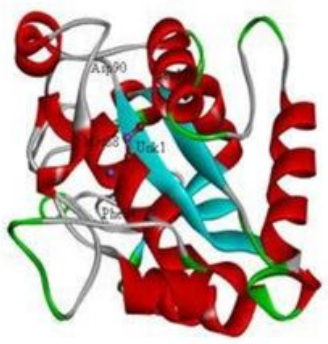

A. annua $\mathrm{PCS}\left(\mathrm{Na}_{2} \mathrm{AsO}_{4}\right)$
(D)

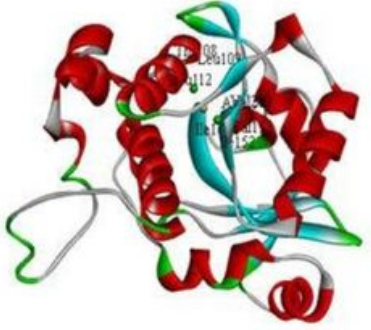

Anabaena PCC $7120 \mathrm{PCS}\left(\mathrm{CdCl}_{2}\right)$
(E)

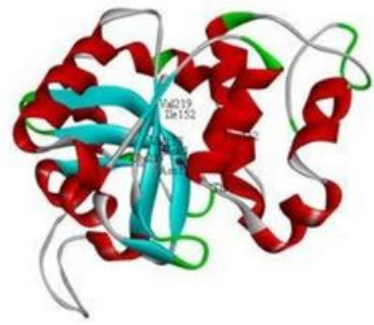

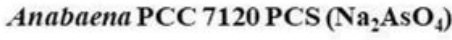

Figure 12 
(A) Comparative analysis of docked complex with experimentally resolved X-RAY diffraction structures of functional domain of AaPCS and anaPCS with cadmium chloride (CdCl2) and sodium arsenate. $(B, C)$ Structure of docked complexes in A. annua as visualized in Discovery Studio 3.0 (D, E) Structure of docked complex in Anabaena PCC 7120 as visualized in Discovery Studio 3.0.

(A)

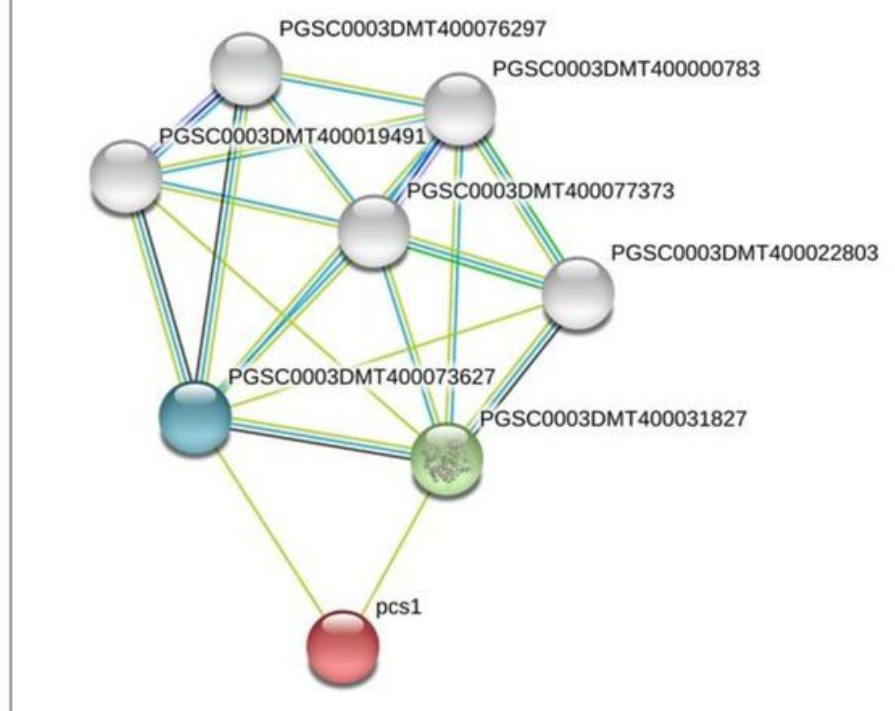

(B)

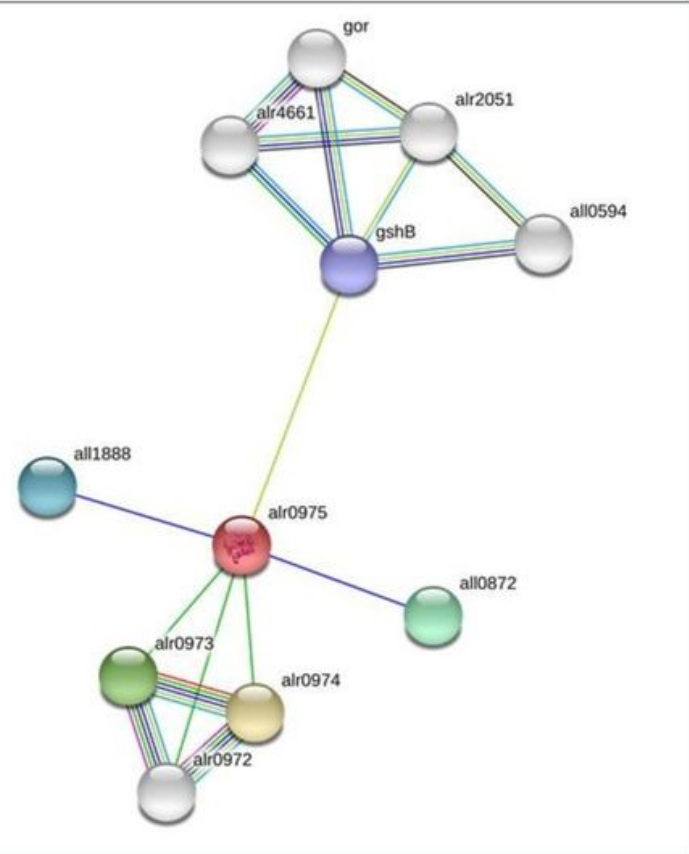

Figure 13

Functional interactive network of (A) AaPCS and (B) anaPCS with other protein family members as found on STRING server where the colored nodes describe query proteins from first shell interactors and white nodes are form second shell interactors. The large node size represents characterized proteins and smaller nodes for uncharacterized proteins.

\section{Supplementary Files}

This is a list of supplementary files associated with this preprint. Click to download.

- Graphicalabstract.jpg

- Supplementarytables.docx

- supplementaryfigures.docx 